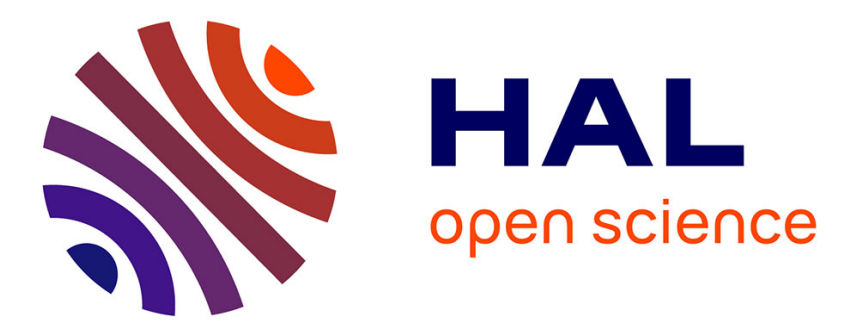

\title{
Interneuronal gamma oscillations in hippocampus via adaptive exponential integrate-and-fire neurons
}

\author{
Liejune Shiau, Laure Buhry
}

\section{To cite this version:}

Liejune Shiau, Laure Buhry. Interneuronal gamma oscillations in hippocampus via adaptive exponential integrate-and-fire neurons. Neurocomputing, 2019, 331, pp.220-234. 10.1016/j.neucom.2018.11.017 . hal-02431627

\section{HAL Id: hal-02431627 \\ https://hal.science/hal-02431627}

Submitted on 14 Jan 2020

HAL is a multi-disciplinary open access archive for the deposit and dissemination of scientific research documents, whether they are published or not. The documents may come from teaching and research institutions in France or abroad, or from public or private research centers.
L'archive ouverte pluridisciplinaire HAL, est destinée au dépôt et à la diffusion de documents scientifiques de niveau recherche, publiés ou non, émanant des établissements d'enseignement et de recherche français ou étrangers, des laboratoires publics ou privés. 


\title{
Interneuronal Gamma Oscillations in Hippocampus via Adaptive Exponential Integrate-and-fire Neurons
}

\author{
LieJune Shiau, Department of Mathematics \\ University of Houston \\ Houston TX 77058, USA \\ Laure Buhry, University of Lorraine, CNRS, Inria,LORIA \\ F-54000 Nancy, France
}

September 30, 2018

\begin{abstract}
Fast neuronal oscillations in gamma frequencies are observed in neocortex and hippocampus during essential arousal behaviors. Through a four-variable Hodgkin-Huxley type model, Wang and Buzsáki have numerically demonstrated that such rhythmic activity can emerge from a random network of GABAergic interneurons via minimum synaptic inputs. In this case, the intrinsic neuronal characteristics and network structure act as the main drive of the rhythm. We investigate inhibitory network synchrony with a low complexity, two-variable adaptive exponential integrate-and-fire (AdEx) model, whose parameters possess strong physiological relevances, and provide a comparison with the two-variable Izhikevich model and Morris-Lecar model. Despite the simplicity of these three models, AdEx model shares two important results with the previous biophysically detailed Hodgkin-Huxley type model: the minimum number of synaptic input necessary to initiate network gamma-band rhythms remains the same, and this number is weakly dependent on the network size. Meanwhile, Izhikevich and Morris-Lecar neurons demonstrate different results in this study. We further investigate the necessary neuronal, synaptic and connectivity properties, including gap junctions and shunting inhibitions, for AdEx model leading to sparse and random network synchrony in gamma rhythms and nested theta gamma rhythms. These findings suggest a computationally more tractable framework for studying synchronized networks in inducing cerebral gamma band activities.
\end{abstract}

\section{Introduction}

Neuronal oscillations of gamma frequency in the brain are known to be related to cognitive functions [23, 40, 53, 52], such as perception [36, 26], attention [18, 12], and memory $[25,34]$. There are various network structures to induce gamma oscillations in the 
brain, and the most fundamental structure and prototype are the interneuronal networks without the involvement of pyramidal neurons. We focus our study on interneuron networks and it is therefore significant to determine the essential intrinsic and synaptic neuron properties that lead to network oscillations in gamma rhythms $[45,53,51,55,56,32]$. The studies of coupled neuronal pairs have provided valuable information in understanding the mechanisms of synchronous oscillations in networks $[51,11,13,14,15]$. Depending on a network architecture, it is possible to predict network synchrony through the information of neuronal pairs. Recent advances in neural anatomy and imaging have opened up new studies in detailed wiring diagrams of functional circuits [8, 35, 39]. Studies of synchronous oscillations with different non-complex network architectures have been considered, and among them, all-to-all globally connected networks and random networks are popularly studied [53]. Studying mutually coupled paired neurons brings insights to all-to-all connected network synchrony [51, 24, 20, 19, 30, 9, 37, 58], but provides little information in random network synchrony.

In addition, it is shown that, in areas of local circuits in hippocampus, synaptic coupling is not all-to-all, and the probability of any pair of cells being connected can be as low as 0.1 to 0.2 [41]. These networks are considered as sparsely and randomly connected $[7,41,42,33]$. Intuitively, it is reasonable to assume that when the connection probability being gradually reduced from 1 (i.e. all-to-all coupling), the network synchrony is to be lost eventually $[1,54,38,4]$. With such a network structure of HodgkinHuxley $(\mathrm{H}-\mathrm{H})$ type neurons, Wang and Buzsáki [53] numerically demonstrate that the minimum number of each synaptic inputs needed to show an initiation of interneuronal network gamma rhythm, approximately $25-90 \mathrm{~Hz}$, is about 60 . Evidently, this minimum number depends on (1) intrinsic and synaptic properties of the neurons, (2) a sufficiently large ratio between the synaptic decay time constant and oscillation period, and (3) a modest heterogeneity of individual neurons. In a similar study, Golomb and Hansel [21] showed theoretically that, in a sparse and random network of identical integrate-and-fire (IF) models, such minimum number is about 360, and also modulated by the intrinsic and synaptic properties of the neurons.

Hence, to study sparse random networks based on results of Wang and Buzsáki [53] and Golomb and Hansel [21], H-H type neurons, instead of IF neurons, would predict gamma network frequencies more easily. However, the dependence of network synchrony on a model type is not clear, and a remaining question is that whether the detailed neuronal physiological properties are necessary to enable an onset network synchrony. We aim to answer this question by studying network behaviors emerged from network structures of interest, and adopting a simple and becoming increasingly popular two-variable adaptive exponential integrate-and-fire (AdEx) model $[3,46,17]$ equipped with a subthreshold adaptation and a spike-triggered adaptation features [49, 32]. More importantly, its parameters resemble salient physiological features and quantities [3, 46], and it has been fitted successfully to approximate the behavior of $\mathrm{H}-\mathrm{H}$ type neurons as well as real recordings from cortical neurons $[3,10,29]$. We compare same network structures of two-variable Izhikevich and Morris-Lecar models, as Izhikevich model [27, 28], exhibits a rich dynamical structure and a wide range of firing patterns as in AdEx model [46, 10], 
and Morris-Lecar model [28, 16], without a reset mechanism, possesses a rather different neuronal feature and oscillatory behaviors from AdEx model. We consider and discuss the effects of gap junctions and shunting inhibitions in studying gamma rhythms, and the generation of nested theta and gamma activities seen in hippocampus.

\section{Models and Methods}

We first study single neurons then network behaviors of AdEx model, including comparisons with networks of Izhikevich and Morris-Lecar models.

\subsection{Networks of AdEx Model}

Neuron model and synaptic connection: Each neuron is described by AdEx neuron, and time evolutions of membrane potential $V$ and current adaptation $w$ are expressed as (more details in $[3,46]$ )

$$
\begin{aligned}
C \frac{d V}{d t} & =-g_{L}\left(V-E_{L}\right)+g_{L} \Delta_{T} e^{\frac{V-V_{T}}{\Delta_{T}}}-I_{\text {shunt }}-w+I_{\text {syn }}(t)+I_{\text {gap }}+I \\
\tau_{w} \frac{d w}{d t} & =a\left(V-E_{L}\right)-w
\end{aligned}
$$

where the evolution of membrane potential depends on a leak term, $g_{L}$ being leak conductance and $E_{L}$ being leak reversal potential, and an exponential spike initiation, $\Delta_{T}$ describing the sharpness of the spike initiation and $V_{T}$ being the onset of membrane potential, followed by a linear coupling with the adaptive current, $w(t), I_{\text {shunt }}=g_{\text {shunt }}\left(V-V_{r}\right)$ is the shunting inhibition component with conductance $g_{\text {shunt }}$ and reset voltage $V_{r}$, $I_{\text {gap }}=g_{\text {gap }}\left(V_{\text {pre }}-V_{\text {post }}\right)$ is the electrical synapse via gap junction with conductance $g_{\text {gap }}$ and membrane potential of pre/post synaptic neuron respectively, and synaptic input is described as $I_{\text {syn }}(t)=-g_{\text {syn }} s(t)\left(V-E_{\text {syn }}\right)$ via a bi-exponential synaptic function

$$
s(t)=c \sum_{t_{j} \leq t}\left(e^{\frac{-\left(t-t_{j}\right)}{\tau_{d}}}-e^{\frac{-\left(t-t_{j}\right)}{\tau_{r}}}\right) .
$$

Here, $g_{s y n}$ is synaptic peak conductance, $E_{\text {syn }}$ is synaptic reversal potential, $\tau_{d}$ and $\tau_{r}$ are synaptic decay and rise time constants respectively, $c$ is a normalization factor chosen to have the peak of $s(t)=1$, and $t_{j}$ is the spike time from neuron $j . I$ is a constant external stimulus bringing membrane potential sufficiently close to or on the spiking region. Whenever the membrane potential reaches threshold $V_{t h}$, the neuron spikes and immediately resets to $V_{r}$ and adaptation current $w$ is increased by a positive amount of $b$ as

$$
\begin{aligned}
& V \rightarrow V_{r} \\
& w \rightarrow w+b
\end{aligned}
$$

As a good comparison with $\mathrm{H}-\mathrm{H}$ type neuron, gap junction and shunting inhibition are not considered until sparse random networks (Section 3.5). 
Parameters: The following parameters are chosen to be close to realistic neurons, $C=0.1 \mathrm{nF}, E_{L}=-70 \mathrm{mV}, \Delta_{T}=2 \mathrm{mV}, V_{r}=-60 \mathrm{mV}, V_{T}=-50 \mathrm{mV}, E_{\text {syn }}=-75$ $\mathrm{mV}, \tau_{r}=0.1 \mathrm{~ms}, \tau_{d}=10 \mathrm{~ms}$, and $V_{t h}=-30 \mathrm{mV}$. For neurons exhibiting type I $f-I$ curve, we have $g_{L}=10 \mathrm{nS}, \tau_{m}=10 \mathrm{~ms}, \tau_{w}=100 \mathrm{~ms}, a=2 \mathrm{nA}, b=4 \mathrm{nA}$, and the total synaptic inputs $g_{\max }=2 \mathrm{nS}$. For neurons exhibiting type II $f-I$ curve, we have $g_{L}=4 \mathrm{nS}, \tau_{m}=25 \mathrm{~ms}, \tau_{w}=25 \mathrm{~ms}, a=12 \mathrm{nA}, b=0 \mathrm{nA}$, and the total synaptic inputs $g_{\max }=32 \mathrm{nS}$. In the following section, we first explain the ranges and other parameters to be chosen to observe a single neuron of frequency about $40 \mathrm{~Hz}$, and network gamma frequency of approximately $30 \mathrm{~Hz}$.

\subsection{Networks of Izhikevich Model}

Neuron model and synaptic connection: Each neuron is described by Izhikevich neuron $[28,45]$, and time evolution is expressed as

$$
\begin{aligned}
\frac{d V}{d t} & =0.04 V^{2}+5 V+140-u+I_{\text {syn }}(t)+I \\
\frac{d u}{d t} & =a(b V-u)
\end{aligned}
$$

where the evolution of membrane voltage is described by a specific dimensionless quadratic polynomial, followed by a linear and dimensionless recovery variable, $u(t)$, and synaptic input function $I_{\text {syn }}(t)$ is the same as in eq. (3). The parameters in Izhikevich model are normalized with limited physiological relevances. When a membrane potential reaches threshold $V_{t h}$, the neuron spikes and immediately resets to $c$ and recovery variable $u$ is increased by $d$, as

$$
\begin{aligned}
& V \rightarrow c \\
& u \rightarrow u+d
\end{aligned}
$$

The notations of the parameters $a, b, c$ and $d$ remain the same as in [27] to reduce confusion. These parameters are dimensionless, and their notations are however irrelevant to those from the AdEx model's.

Parameters: With same values of $V_{t h}=-30, \tau_{r}=0.1$ and $\tau_{d}=10$ as in AdEx model, the following parameter values are chosen. For neurons of type I $f-I$ curve, we have $a=0.02, b=-0.1, c=-65, d=6, I=34$ and $g_{\max }=6$. For neurons of type II $f-I$ curve, we have $a=0.1, b=0.26, c=-60, d=-1, I=15$ and $g_{\text {max }}=2$. 


\subsection{Networks of Morris-Lecar Model}

Neuron model and synaptic connection: Each neuron is described by Morris-Lecar neuron $[28,16]$, and time evolution is expressed as

$$
\begin{aligned}
C \frac{d V}{d t} & =-g_{L}\left(V-E_{L}\right)-g_{K} n\left(V-E_{K}\right)-g_{C a} m_{\infty}(V)\left(V-E_{C a}\right)+I_{\text {syn }}(t)+I \\
\frac{d n}{d t} & =\phi\left(n_{\infty}(V)-n\right) / \tau_{n}(V)
\end{aligned}
$$

Where the evolution of membrane voltage is described by a leak, a potassium channel and a calcium channel, and coupled with a potassium gating variable. Further more, $m_{\infty}(V)=\frac{1}{2}\left[1+\tanh \left(\frac{V-V_{1}}{V_{2}}\right)\right], n_{\infty}(V)=\frac{1}{2}\left[1+\tanh \left(\frac{V-V_{3}}{V_{4}}\right)\right]$, and $\tau_{n}(V)=1 / \cosh \frac{V-V_{3}}{2 V_{4}}$, where parameters $V_{1}, V_{2}, V_{3}$, and $V_{4}$ are chosen to fit voltage-clamp data, $\phi$ is a constant, and the synaptic input $I_{\text {syn }}(t)$ is the same as in eq. (3).

Parameters: With same time constants $\tau_{r}=0.1 \mathrm{~ms}$ and $\tau_{d}=10 \mathrm{~ms}$ as in AdEx model, the following parameter values are chosen. $C=20 \mu \mathrm{F} / \mathrm{cm}^{2}, V_{1}=-1.2 \mathrm{mV}$, $V_{2}=18 \mathrm{mV}, V_{3}=12 \mathrm{mV}, V_{4}=17.4 \mathrm{mV}, g_{C a}=6 \mathrm{mS} / \mathrm{cm}^{2}, g_{K}=30 \mathrm{mS} / \mathrm{cm}^{2}, g_{L}=2$ $\mathrm{mS} / \mathrm{cm}^{2}, E_{C a}=120 \mathrm{mV}, E_{K}=-90 \mathrm{mV}, E_{L}=-65 \mathrm{mV}, \phi=1 . / 15, I=110 \mu \mathrm{A}$.

\subsection{Methods}

Random network connections: For networks of total $N$ neurons, the directional coupling between a pair of neurons is randomly assigned with a probability $p$. The all-to-all coupled networks are with a probability $p=1$ between each pair of neurons. In this study, maximum synaptic conductance $g_{\text {syn }}$ is divided by the number of synaptic inputs $M_{\text {syn }}=N \cdot p$. Therefore, the number of synaptic inputs $M_{\text {syn }}$ may vary, but, on average, the total synaptic drive per neuron remains the same. Nevertheless, we demonstrate that $M_{\text {syn }}$ plays an important role in network behaviors.

A measure of network synchrony: To quantify the degree of network synchrony, we adopt a network coherence index as a measurement, and it is defined based on a coherence index between synchrony of each spiking pairs in a weak sense [53]. A coherence index between two neurons $i$ and $j$ is measured by their normalized cross-correlation of spike trains at zero time lag within each time bin of $\tau$ over a time interval of the firing period $T$, i.e. $\tau \in[0, T]$. More specifically, when two spike trains are given by $X(l)=0$ or 1 , and $Y(l)=0$ or $1, l=1,2, \cdots, K$, with $K=T / \tau$, we define a coherence index for the pair of neurons as

$$
\kappa_{i j}(\tau)=\frac{\sum_{l=1}^{K} X(l) Y(l)}{\sqrt{\sum_{l=1}^{K} X(l) \sum_{l=1}^{K} Y(l)}}
$$

The coherence index of a network of neurons, $\kappa(\tau)$, is defined as the average of $\kappa_{i j}(\tau)$ over all discrete pairs of neuron in the network of $N$ neurons, with $i<j$ for all $i, j \in N$, and it provides information about neuronal interactions and network synchrony. A network coherence index $\kappa(\tau)$ is calculated by averaging over a subset of discrete neuronal 
pairs that are drawn randomly from all possible combinations in the network.

A measure of network frequency: Network frequency is computed as the average frequency of all neuronal frequencies.

Numerical simulations: Network simulations are performed through Brian 2 software developed by Romain Brette, et al. [22] and Marcel Stimberg, et al. [43], released under the CeCILL license. Plotting of the results is performed in Python 2.7. The simulations are done with a time step of $0.01 \mathrm{~ms}$, coherence index is calculated after discarding the first $500 \mathrm{~ms}$ transients, and time bin is chosen for $1 \mathrm{~ms}$ with single neuronal frequency about $40 \mathrm{~Hz}$ and network synchrony about $30 \mathrm{~Hz}$ as in [53] for a good comparison.

Organization of the results: Firstly we identify the intrinsic characteristics in single AdEx neurons that lead to network oscillations within gamma rhythms. Based on these characteristics, we next determine other intrinsic properties necessary to induce network synchrony first for identical AdEx neurons then followed by heterogeneous AdEx neurons in all-to-all coupled networks. The next step is to study synchrony of heterogeneous AdEx neurons in randomly connected networks, and to demonstrate the minimum number of synaptic connections needed in each neuron to initiate network synchrony. We then demonstrate the minimum number of synaptic connections needed per neuron under the same network structure through Izhikevich and Morris-Lecar neurons. Lastly, for a sparsely and randomly connected network of heterogeneous AdEx neurons, with a high degree of network synchrony for frequency about $30 \mathrm{~Hz}$, we demonstrate the ranges of parameters necessary to achieve such performance. We also discuss the inclusion of gap junctions and shunting inhibitions in such networks to induce gamma rhythms, and their roles in impacting network synchrony and frequency. Finally, we validate the network gamma rhythms with experimental recording data from rodent, and further consider the more complex theta-nested gamma oscillations that may be generated via AdEx models.

\section{Results}

\subsection{Single neurons to a population of uncoupled heterogenous neurons}

We first investigate the distinct intrinsic neuronal adaptation properties, $a$ and $b$, of AdEx neurons, and show how they impact neuronal/network frequency leading to gamma rhythms.

Intrinsic neuronal properties It is essential to understand all-to-all network behaviors in order to gain insights in studying special networks. We first identify the intrinsic characteristics in single AdEx neurons that lead to oscillations with gamma rhythms. The most distinct characteristics of AdEx neuron is the adaptation property: 
subthreshold adaptation $a$ and spike-triggered adaptation $b$ (eq. (1), (4)). Their $f-I$ curves, frequency response to input current functions, are shown in (Fig. 1). For a fixed
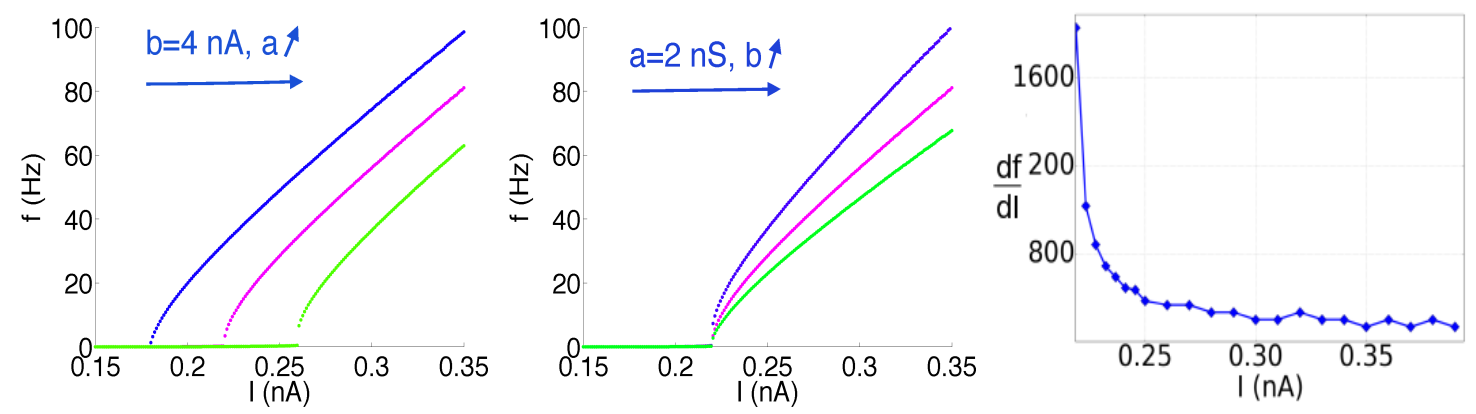

Figure 1: frequency vs applied current curves from left to right are (left) $a=0,2,4$ (nS) (with $b=4 \mathrm{nA}$ ); (middle) $b=0,4,8$ (nA) (with $a=2 \mathrm{nS}$ ). Both indicate frequency slows down, as $a$ or $b$ increases. (right) $d f / d I$ curve illustrates that the slope of frequency curve is steep at low current input, and becomes moderate showing frequency slowing down $(a=2 \mathrm{nS}, b=4 \mathrm{pA})$.

current $I$, as adaptation $a$ (or $b$ ) increases (Fig. 1 (left) or (middle)), the firing frequency decreases accordingly. Both figures show an initial nonlinear sharp frequency increase, then slowed down and followed by a nearly linear growth. The derivative of $f-I$ curve (Fig. 1 (right)) illustrates this property. Hence, the adaptations should be relatively moderate in order to maintain gamma rhythms. We take subthreshold adaptation $a=2$ $\mathrm{nS}$, spike-triggered adaptation $b=4 \mathrm{nA}$, and input current $I=0.25 \mathrm{nA}$ in single AdEx neurons to study network gamma rhythms about $30 \mathrm{~Hz}$.

Collective frequency of a population of uncoupled heterogeneous neurons In order to understand the sensitivity of heterogenous input in relation to collective population frequency, we simulate a population of 100 uncoupled heterogeneous neurons, each receiving an input current drawn from Gaussian distribution with mean $I_{\mu}$ and standard deviation $I_{\sigma}$. By varying mean input current $I_{\mu}$ with a fixed fluctuation $I_{\sigma}=$ $0.3 \mathrm{pA}$, we show that the relative fluctuation of population frequency, $f_{\sigma} / f_{\mu}$, is slightly larger at low applied current (near rheobase) (Fig. 2 (left)) (or at low population mean frequency (Fig. 2 (right))) but is generally very small, implying less population frequency fluctuations to be observed at slightly higher $I_{\mu}$ or $f_{\mu}$.

\subsection{Synaptic characteristics and all-to-all coupled networks}

We now demonstrate the necessary synaptic characteristics to induce network frequency in all-to-all coupled identical neurons.

Synaptic characteristics: We show that, in simulating AMPA-mediated type excitatory coupling networks with parameters to maintain $30 \mathrm{~Hz}$ frequency, networks fail to synchronize and neurons fire essentially arbitrarily (Fig. 3 (left)). Particularly, network coherence index $\kappa(\tau)$ increases linearly with time bin size $\tau \in[0, T]$ from 0 to 1 

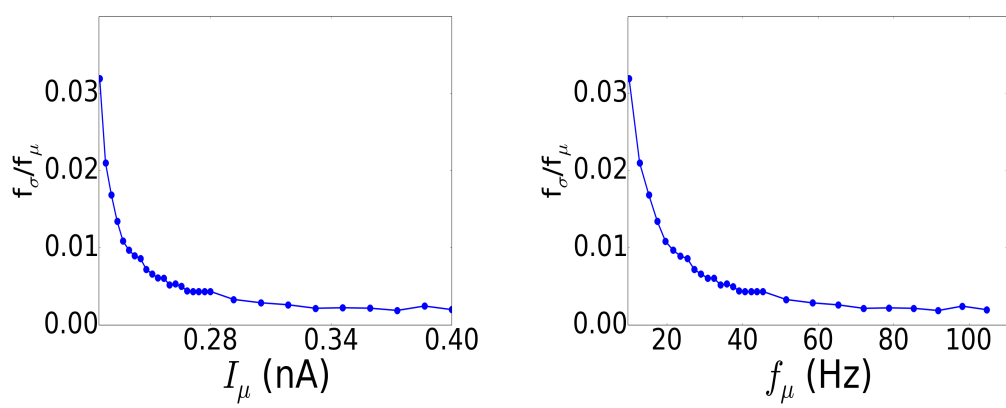

Figure 2: Both coefficient of variation, $f_{\sigma} / f_{\mu}$, plots are relatively similar, despite that the plots are against mean input current $I_{\mu}$ (left) or mean population frequency $f_{\mu}$ (right). It demonstrates that frequency fluctuation is reduced by stronger mean input current $I_{\mu}$ (left) and by higher mean population frequency $f_{\mu}$.

(Fig. 3 (right)), showing that the relative firing time of neural pairs is nearly uniformly distributed between 0 and the period of firing $T$ indicating network asynchrony.
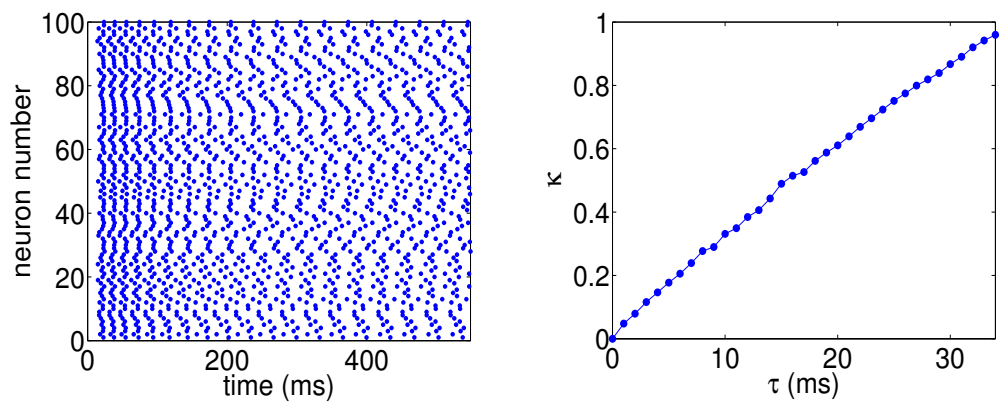

Figure 3: (left) Raster plot shows network asynchronous (right) Network coherence index increases linearly with time bin size. It is a signature of network asynchrony. $\left(E_{\text {syn }}=0\right.$ $\left.\mathrm{mV}, \tau_{r}=0.1 \mathrm{~ms}, \tau_{d}=5 \mathrm{~ms}, I=0.235 \mathrm{nA}, a=2 \mathrm{nA}, b=4 \mathrm{nS}, I=0.25\right)$

Network synchrony and synaptic potential: We next examine the degree of network synchrony with inhibitory synaptic coupling, such as in GABAergic interneurons, and all the intrinsic cell properties remained, through a network of $N=100$ identical neurons with all-to-all coupling, without neuronal heterogeneity. We demonstrate that network synchrony is greatly affected by the level of the synaptic reversal potential $E_{\text {syn }}$. With a low $E_{\text {syn }}=-90 \mathrm{mV}$, the network synchronizes quickly after several spikes (Fig. 4 (left)). As $E_{\text {syn }}$ increases to near $V_{r}=-60 \mathrm{mV}$, such as $E_{\text {syn }}=-75 \mathrm{mV}$, the network synchronizes after a longer period of transients (Fig. 4 (middle)). While $E_{\text {syn }}$ increases and passes $V_{r}$, such as $E_{\text {syn }}=-40 \mathrm{mV}$, network synchrony deteriorates rapidly and leads to asynchrony (Fig. 4 (right)). The relationship between intrinsic and synaptic properties of minimum membrane voltage remaining above the synaptic reversal potential $E_{\text {syn }}$ is found to be an important condition leading to network coherent synchrony. Fig. 5 demonstrates how network synchrony is affected by synaptic reversal potential $E_{\text {syn }}$. It indicates a gradual decrease in network synchrony, as $E_{\text {syn }}$ approaches $V_{r}$. It 

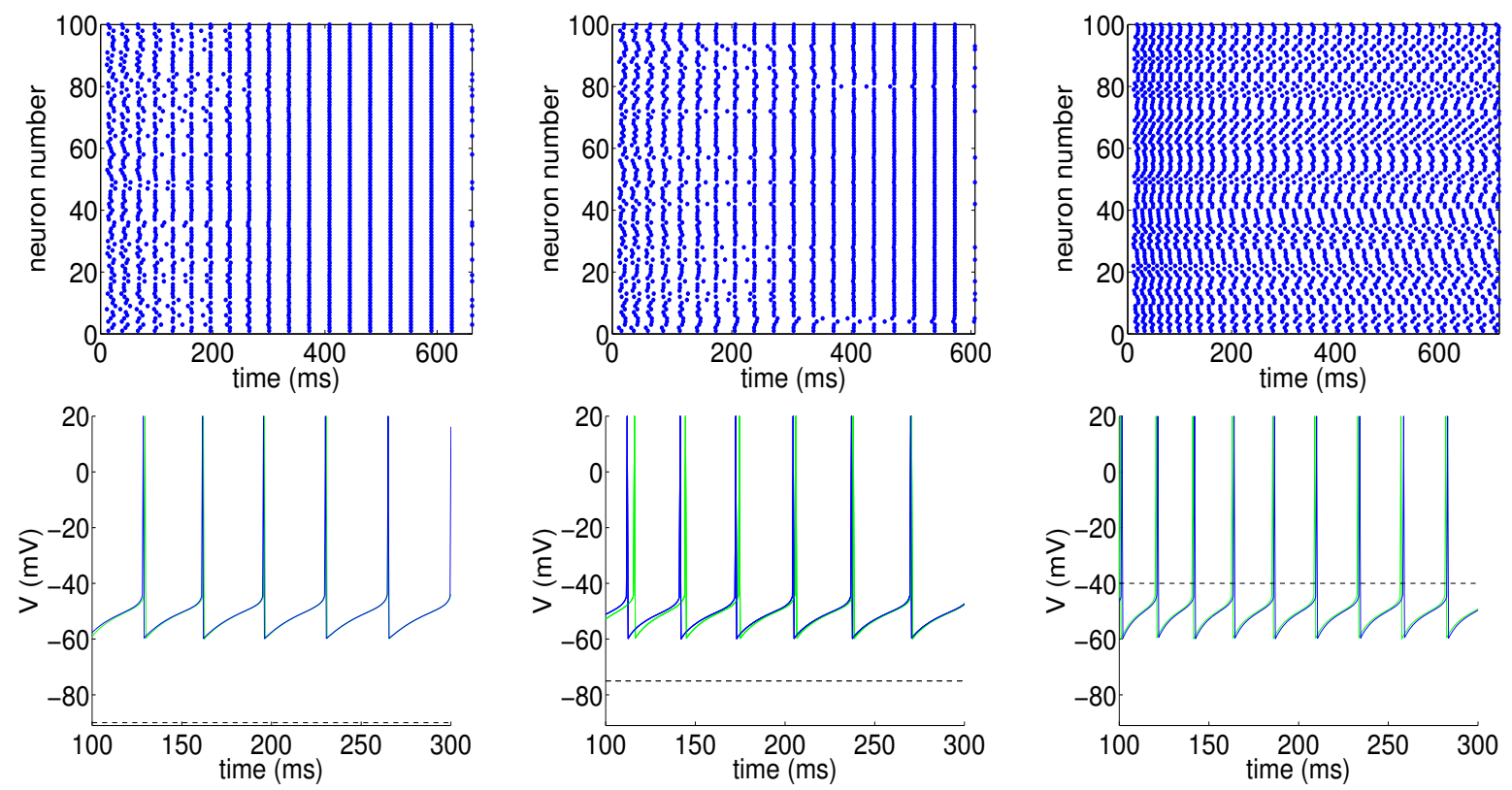

Figure 4: (top) raster plots (bottom) membrane potentials of two arbitrary neurons (blue and green) from the network, with the value of $E_{\text {syn }}$ marked in dotted line. (left) $E_{\text {syn }}=-90 \mathrm{mV}$ (middle) $E_{\text {syn }}=-75 \mathrm{mV}$ (right) $E_{\text {syn }}=-40 \mathrm{mV}(I=0.25 \mathrm{nA}$, network frequency $\approx 30 \mathrm{~Hz}$.)

then followed by a rapid decrease, as $E_{\text {syn }}$ passes $V_{r}$. Compared to the abrupt decrease of $\mathrm{H}-\mathrm{H}$ type neural networks [53], network synchrony of AdEx neurons deteriorates more smoothly near the critical value of $E_{\text {syn }}$. Hence, $E_{\text {syn }}=-75 \mathrm{mV}$ is taken in remaining simulations to ensure that the effect of synaptic inputs is hyperpolarizing to achieve some degree of network synchrony.

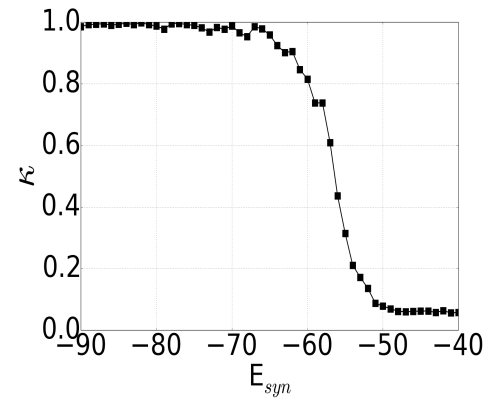

Figure 5: As $E_{\text {syn }}$ increases, $\kappa$ decreases first gradually then sharply around $V_{r}=-60$ $\mathrm{mV}$ followed by asynchronous networks. $(I=0.25 \mathrm{nA}$.)

Network synchrony and time constants: We demonstrate how time constants, such as intrinsic adaptation time constant $\tau_{w}$, and synaptic rise and decay time constants $\tau_{r}$ and $\tau_{d}$, influence the coupling behaviors in leading to different degrees of network synchrony. Plots in (Fig. 6 (left)) show that stronger $\tau_{w}$ slows down network frequency 
but only reduces network synchrony slightly. On the other hand, within a range of synaptic rise time constant $\tau_{r}$, network frequency and synchrony are nearly preserved (Fig. 6 (middle)). (Fig. 6 (right)) shows that as $\tau_{d}$ increases, network synchrony is low initially, due to neuronal phase locking. As $\tau_{d}$ continues to increase, the phase locking difference decreases. Once the phase locking difference becomes less than the time bin for calculating $\kappa$, it shows a sudden increase of network synchrony $\kappa$. This reflects on the sudden jumps of network frequency and network coherence index near $\tau_{d}=4$ ms. These indicate the significant role played by the synaptic decay time constant in network synchrony, while the role of synaptic rising time constant is less influential under such conditions. To achieve network synchrony with frequency approximately $30 \mathrm{~Hz}$, $\tau_{w}=100 \mathrm{~ms}, \tau_{r}=0.1 \mathrm{~ms}$, and $\tau_{d}=10 \mathrm{~ms}$ are taken thereafter.
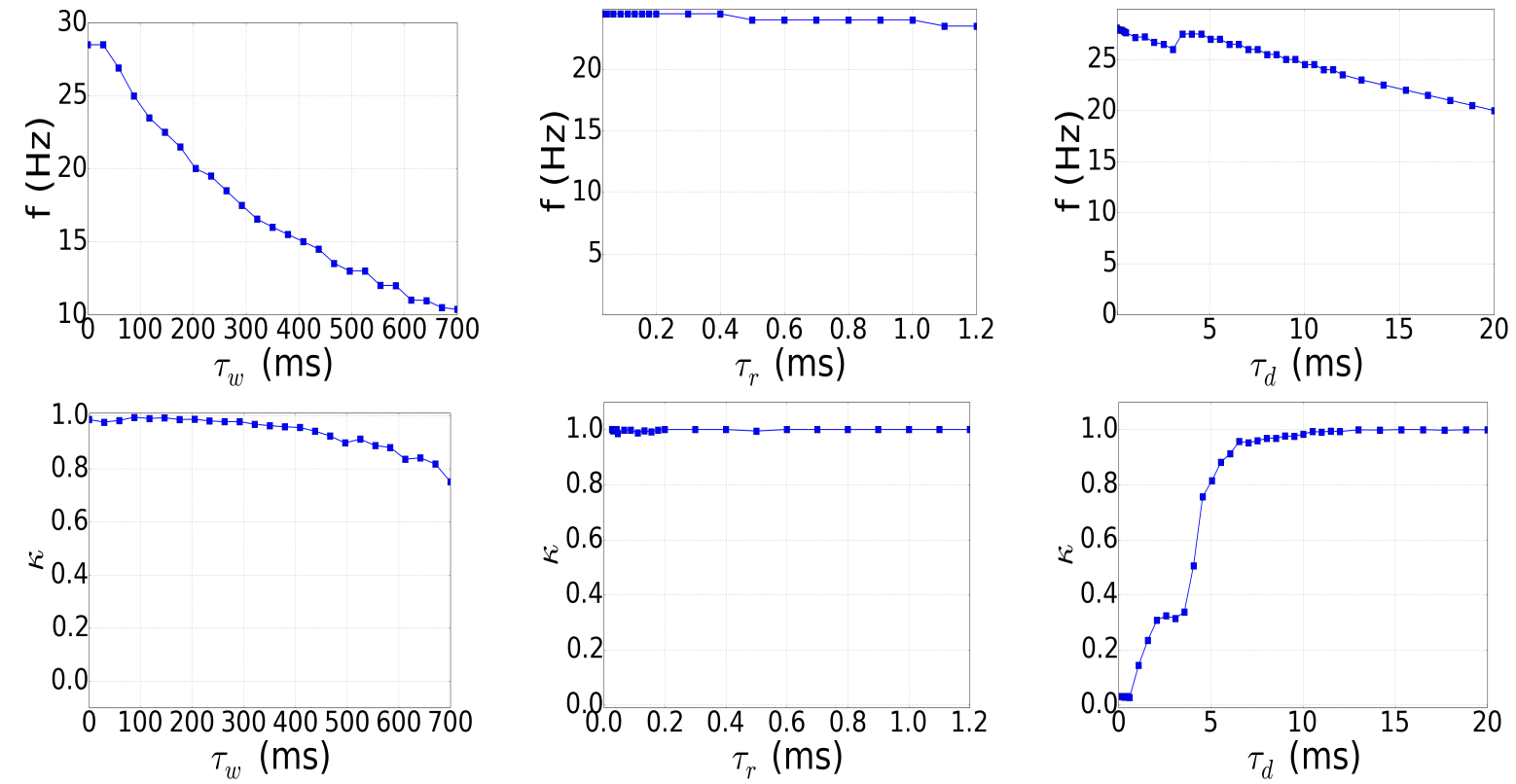

Figure 6: Network frequency/synchrony ((left) top/bottom) decreases, as $\tau_{w}$ increases; ((middle) top/bottom) persists, as $\tau_{r}$ increases; ((right) top/bottom) decreases/increases, as $\tau_{d}$ increases. A fast synaptic decay time constant leads to a low degree of network synchrony, while a slow synaptic decay time constant helps to promote network synchrony. ( $I=0.25 \mathrm{nA})$

Network synchrony and adaptations: We demonstrate how network synchrony changes with the variations of subthreshold adaptation $a$ and spike-triggered adaptation $b$. It is shown that if $a>g_{L} \frac{\tau_{m}}{\tau_{w}}$, the system undergoes subcritical Hopf bifurcation [46]. The neuron no longer fires, once the subthreshold adaptation parameter a passes a critical value $a_{c}$ (leading to subcritical Hopf bifurcation) which can be calculated as

$$
a_{c}=g_{L}\left(\frac{1-\frac{\tau_{m}}{\tau_{w}}+\frac{I}{\Delta_{T} g_{L}}}{\ln \left(1-\frac{\tau_{m}}{\tau_{w}}\right)-\frac{E_{L}-V_{T}}{\Delta_{T}}}-1\right)
$$


This indicates that the value of $a$ is limited by $a_{c}$ for firing neurons (Fig. 7 (left)), while $b$ (independent of bifurcation) can have a broader range of choices (Fig. 7 (right)) to maintain neuronal firings. We now examine the way $a$ affects the associated coherence
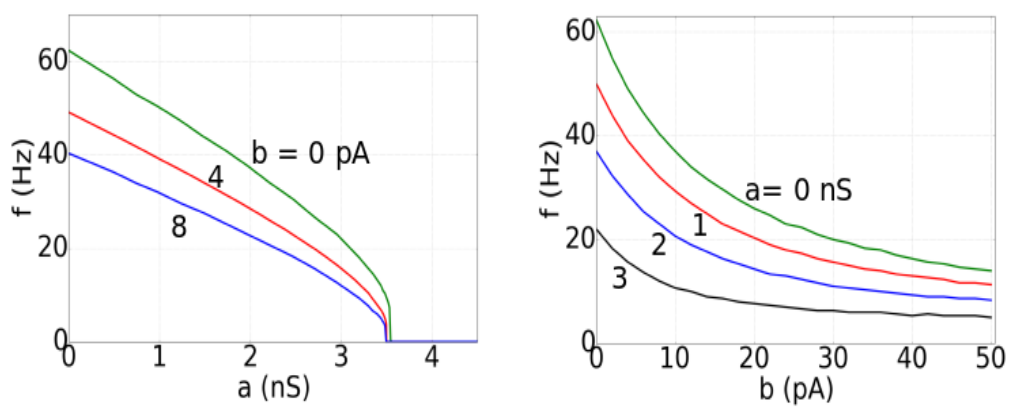

Figure 7: frequency plots: (left) $a$ has a maximumvalue to elicit spikes. Once $a>$ $a_{c}=3.54 \mathrm{nS}$, neuronal firing is lost. (right) $b$ can continue to grow and spike frequency continues to drop down towards zero. $(I=0.25 \mathrm{nA})$

index $\kappa(\tau)$ in such networks accordingly (Fig. 8 (left)). The subthreshold adaptation $a$ needs to be relatively modest and away from the critical value $a_{c}=3.54 \mathrm{nS}$ to achieve network synchrony in these networks. We choose $a=2 \mathrm{nS}$ in our network simulations. The way spike-triggered adaptation $b$ affects the associated coherence index $\kappa(\tau)$ over
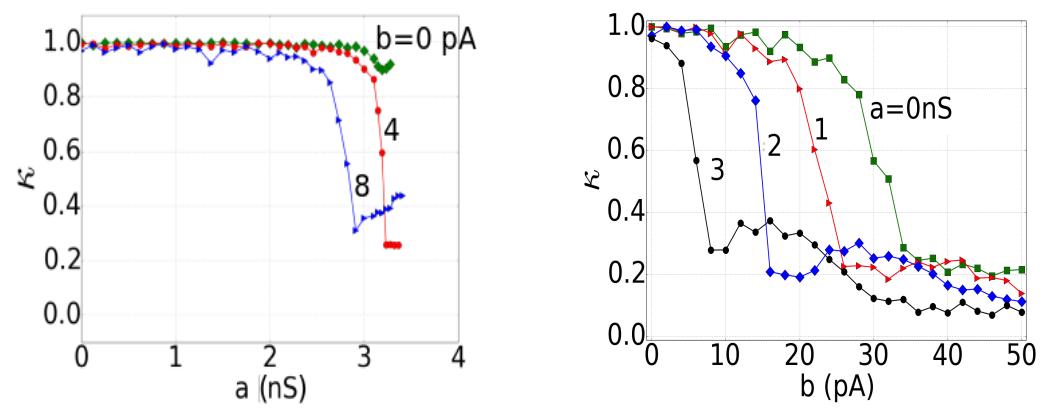

Figure 8: Network synchrony plots: Networks become less synchronous then become asynchronous, as $a$ (left) or $b$ (right) increases.

various values of $a$ is shown in (Fig. 8(right)). For $a=2 \mathrm{nS}$, in order to maintain a high degree of network synchrony, the spike-triggered adaptation $b$ needs to be no less than $6 \mathrm{nA}$. Hence, $b=4 \mathrm{nA}$ is taken in our network simulations.

\subsection{Network synchrony of heterogeneous neurons via random coupling}

We have demonstrated that network synchrony can be achieved under all-to-all inhibitory network of identical AdEx neurons to exhibit gamma rhythms. This is interneuronal network gamma rhythm, as one of the fundamental structures of generating gamma frequencies $[44,45,60,59]$. It is expected that network synchrony will deteriorate 
if the differences among neuronal frequencies become greater, or the number of synaptic coupling is decreased in each neuron. We start with networks of heterogeneous neurons, via an all-to-all coupling, to observe the way that individual neural frequencies affect their network synchrony, then we study randomly connected networks to demonstrate the minimum coupling number needed in each neuron to observe the onset of network synchrony.

\subsubsection{All-to-all coupled network synchrony of heterogeneous neurons}

We demonstrate the effects of network heterogeneity on network behaviors, under the applied current with amplitudes drawn from a Gaussian distribution with a mean of $I_{\mu}$ and a standard deviation of $I_{\sigma}$. As demonstrated (Fig. 9 (left)), network synchrony decreases sharply, as the variation of input currents begins to widen approaching to $I_{\sigma}=0.3 \mathrm{pA}$. It continues to decrease and eventually loses any synchrony as the variation of input current increases approaching to $I_{\sigma}=2 \mathrm{pA}$. (Fig. 9 (right)) indicates that, with small dispersions in current input, the average of network frequency $f_{\mu}$ fluctuates slightly. As the dispersion of input current begins to increase furthermore, the dispersion of individual neuron frequency $f_{\sigma}$ also increases while the average of network frequency $f_{\mu}$ decreases slightly.
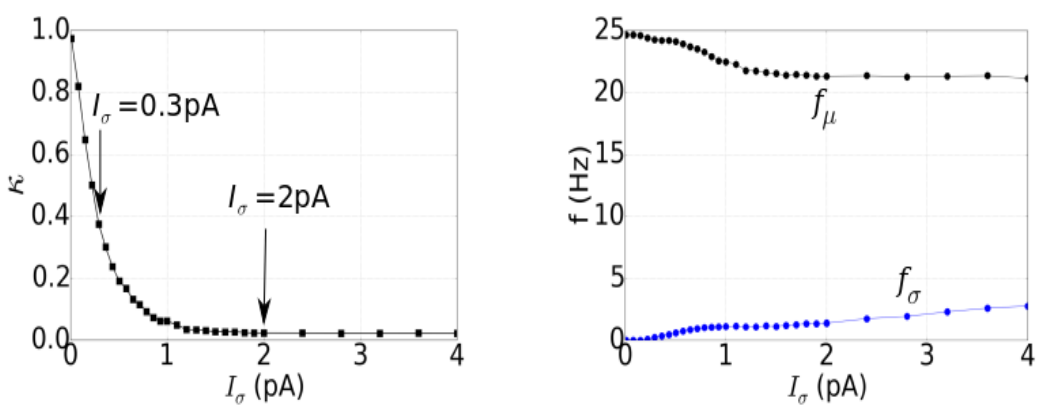

Figure 9: (left) network synchrony $\kappa$ vs heterogeneity $I_{\sigma}$ plot: It shows that $\kappa$ deteriorates, as neurons become more heterogeneous. (right) network frequency $f$ vs heterogeneity $I_{\sigma}$ plot: It indicates $f_{\mu}$ deteriorates while $f_{\sigma}$ increases, as neurons become more heterogeneous.

This kind of dispersion increase in each neuron and decrease in the network frequencies for AdEx neurons are relatively mild, as supposed to those of $\mathrm{H}-\mathrm{H}$ type of neurons being more pronounced [53]. Associated with the parameters marked as the left arrow in (Fig. 9 (left)), we demonstrate that network synchrony $\kappa$ also depends on the time bin size $\tau$ chosen. With $I_{\sigma}=0.3 \mathrm{nA}$, (Fig. 10 (top left)) illustrates a partial network synchrony with $\kappa$ approximately to be 0.4 (with $\tau=2 \mathrm{~ms}$ ). High nonlinearity of the curve (Fig. 10 (top right)) indicates the network coherence index increases for the time bin size from 1 to $5 \mathrm{~ms}$. That shows partial network synchrony can be achieved within a limited range of small time bin $\tau$. Once the time bin size reaches approximately $\tau=10 \mathrm{~ms}$, the full network synchrony can be achieved accordingly. On the contrary, 
associated with the parameters marked as the right arrow in (Fig. 9 (left)), for the larger input dispersion $I_{\sigma}=2 \mathrm{pA}$, the raster plot (Fig. 10 (bottom left)) shows an asynchronous network with no sign of synchrony. The linearity of network synchrony curve indicates network asynchrony. These results imply that to observe some degree of network synchrony in all-to-all networks of heterogeneous neurons, the input dispersion $I_{\sigma}$ should remain relatively moderate. We will take $I_{\sigma}=0.3 \mathrm{pA}$ to allow a range of heterogeneous neurons, as we progress to networks of heterogeneous neurons with a lower degree of connectivity than all-to-all in the next discussion.
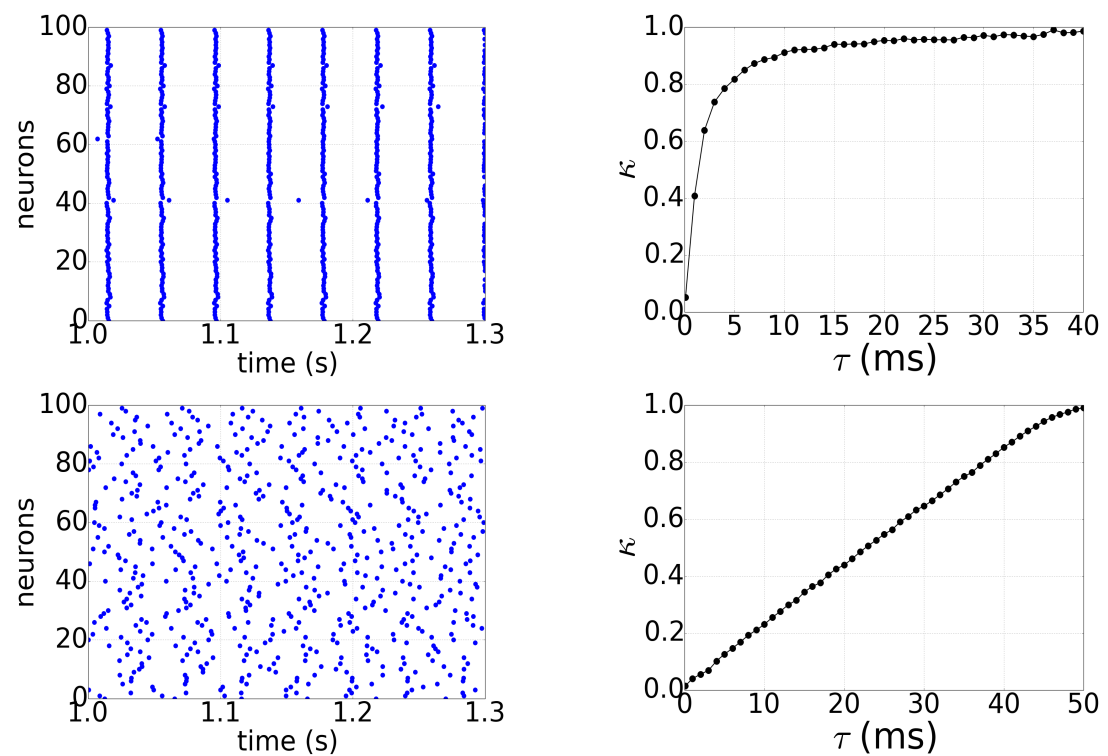

Figure 10: (upper left) Raster plot shows partial network synchrony of $\kappa=0.4\left(I_{\sigma}=0.3\right.$ pA). (upper right) Network synchrony vs time bin plot confirms the partial synchrony. (lower left) Raster plot shows network asynchrony $\left(I_{\sigma}=2 \mathrm{pA}\right)$. (lower right) The linearity of network synchrony curve validates network asynchrony. $\left(I_{\mu}=0.25 \mathrm{nA}\right)$

\subsubsection{Minimum connections in randomly connected heterogeneous networks}

We now discuss networks of heterogeneous neurons reducing all-to-all coupling to random connections with a constant number of coupling per neuron on average. Random connections in a network can be considered as a neuron making synaptic contact to a second neuron with a probability $p$. That is, if $N$ is the total number of neurons, then there are $M_{\text {syn }}=p N$ pre-synaptic neurons that converge to a post-synaptic neuron on average. (We consider that the sum of total synaptic inputs into each neuron remains the same, and it is divided into $M_{\text {syn }}$ pre-synaptic neurons.) It is shown previously that networks can synchronize through all-to-all connectivity $\left(M_{s y n}=N, p=1\right)$ with proper choices of synaptic reversal potential $E_{\text {syn }}$, subthreshold adaptation parameter $a$, spiketriggered adaptation parameter $b$, adaptive current time constant $\tau_{w}$, along with the time constants for synaptic rise $\tau_{r}$ and decay $\tau_{d}$. On the contrary, it is easy to see that 
without any connections $\left(M_{\text {syn }}=0\right)$, the networks cannot achieve synchrony. We aim to identify $M_{s y n}^{\min }$, the minimum connections necessary to observe the onset of network synchrony, say $\kappa \approx 0.05$, in randomly connected networks. In Fig. 11 (left), we demonstrate
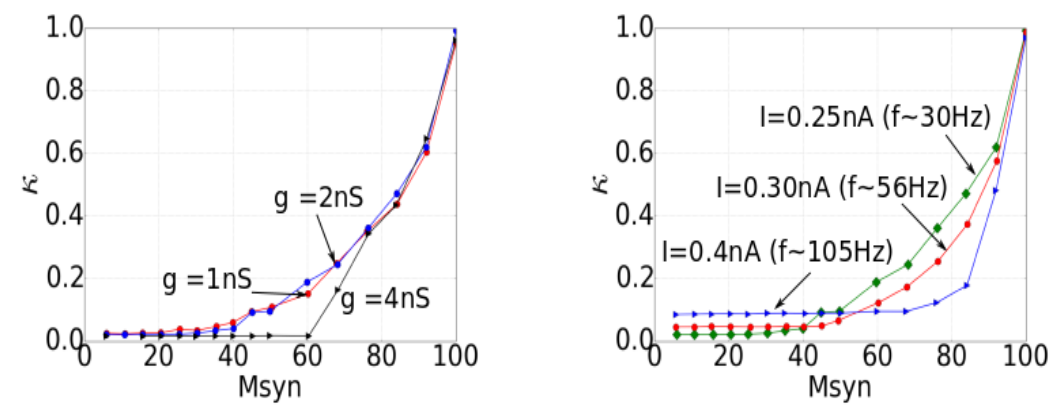

Figure 11: $\kappa$ vs $M_{\text {syn }}$ plots for networks of $N=100$ neurons. (left) For $g_{\text {syn }}=1$ or $2 \mathrm{nS}$, $M_{\text {syn }}^{\min } \approx 60$, and for $g_{\text {syn }}=4 \mathrm{nS}, M_{\text {syn }}^{\text {min }}$ is higher. $(I=0.25 \mathrm{pA})$ (right) As the current $I$ increases moderately (in maintaining low gamma rhythms), $M_{\text {syn }}^{\text {min }}$ remains about 60 . For $I=0.4 \mathrm{nA}$, network frequency is above gamma oscillations $\left(g_{\text {syn }}=2 \mathrm{nS}\right)$.

that $M_{s y n}^{\min }$, the minimum connections necessary to show the onset of network synchrony are approximately 60 for synaptic conductance $g_{\text {syn }}=1$ and $2 \mathrm{nS}$, and a slightly higher minimum number for a stronger synaptic conductance of $g_{\text {syn }}=4 \mathrm{nS}$. In Fig. 11 (right), we illustrate that though input current can also affect $M_{s y n}^{\min }$, a moderate range of input current affects it very weakly. Similarly, for response frequencies between 30 to $60 \mathrm{~Hz}$, an onset of network synchrony is also about 60 minimum connections per neuron.
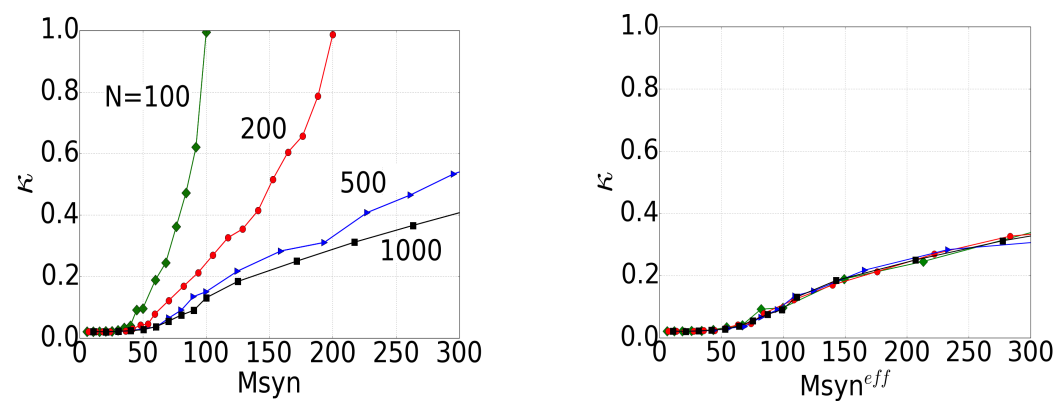

Figure 12: $\kappa$ vs $M_{\text {syn }}$ and $M_{\text {syn }}^{\text {eff }}$ plots for networks of $N$ neurons. (left) The onset of network synchrony of each curve is about 60 . That is, $M_{s y n}^{\min } \approx 60$ which is relatively insensitive to the network size $N$. (right) All normalized curves become one which remains flat until $M_{\text {syn }}^{\text {eff }}$ crossing $M_{\text {syn }}^{\min }$ connections $\left(I=0.25, g_{\text {syn }}=2 \mathrm{nS}\right)$.

A significant result from Fig. 12 (left) shows that $M_{s y n}^{\min }$ is about 60 for $N=100,200,500$, or 1000 , with sum of $g_{\text {syn }}=2 \mathrm{nS}$ per neuron while maintaining network frequency about $30 \mathrm{~Hz}$. In this case, $M_{s y n}^{\min }$ remains small and is not a fraction of the network size $N$. 
$M_{s y n}^{\min }$ only weakly depends on $N$. This result is surprisingly similar to $\mathrm{H}-\mathrm{H}$ type neurons [53].

Let $M_{s y n}^{e f f}$ be the effectiveness of $M_{s y n}$. It can be formulated as

$$
\frac{1}{M_{s y n}^{e f f}}=\frac{1}{M_{s y n}}-\frac{1}{N}
$$

We demonstrate that all curves from (Fig. 12 (left)) converge into one normalized curve through the effectiveness of the minimum number of connections (Fig. 12 (right)), and the nonlinearity of this curve can be observed once $M_{s y n}^{e f f}$ reaches about 60 . This indicates that, to achieve a higher degree of network synchrony beyond the onset, $M_{s y n}^{\min }$ is weakly depending on network size $N$.

$\kappa$ versus $M_{\text {syn }}$ curves as parameter varies: Though most parameters are kept constant while measuring network synchrony on $M_{s y n}$ (and $M_{s y n}^{e f f}$ ), the results are relatively similar while most parameters varying within gamma rhythms. For instance, if $E_{\text {syn }}=-85 \mathrm{mV}$ (similarly for $a=1 \mathrm{nS}$ or $b=6 \mathrm{nA}$ is taken respectively), network synchrony responses on $M_{\text {syn }}$ shown in (Fig. 13 (left)) are nearly identical with those in (Fig. 12 (left)). Results of network synchrony responses on $M_{s y n}^{\text {eff }}$ (not shown) are also the same as those in (Fig. 12 (right)). If $\tau_{w}=50 \mathrm{~ms}$ is taken, network index responses (Fig. 13 (middle)) are nearly identical with those in (Fig. 12 (left)), except when $N=1000$. The reason is when intrinsic current becomes much faster (compared to $\tau_{w}=100 \mathrm{~ms}$ in (Fig. 12 (left))) in low connectivity networks (for $N=1000$ up to $M_{\text {syn }}=300$ in the plot), network synchrony is more difficult to achieve. To main gamma oscillations, synaptic rise time constant $\tau_{r}$ needs to be small (see (Fig. 6)). Varying $\tau_{r}$ within a reasonable range does not affect network coherent index leading to same network index responses on $M_{\text {syn }}$ as in (Fig. 12 (left)). If a faster synaptic decay time constant $\tau_{d}=5 \mathrm{~ms}$ is taken (compared to $\tau_{d}=10 \mathrm{~ms}$ in (Fig. 12 (left))), network sychrony is expected to decrease (see (Fig. 6)) on $M_{\text {syn }}$ as in (Fig. 13 (right)) and a full-scale synchrony fails $(\kappa \neq 1)$. The less than smooth appearance on the curves is due to the random connectivity on the networks.
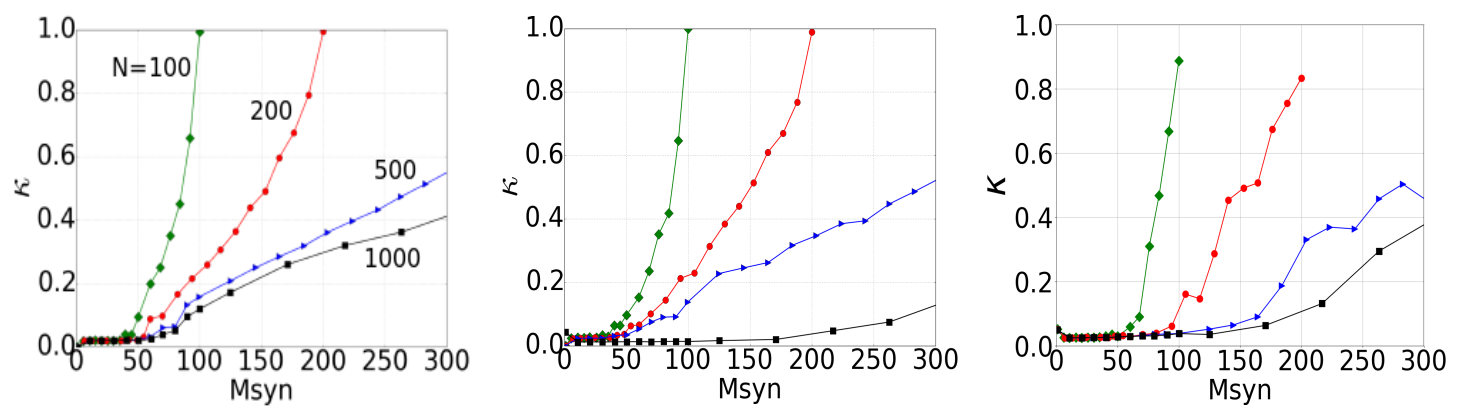

Figure 13: $\kappa$ vs $M_{\text {syn }}:$ (left) $E_{\text {syn }}=-85 \mathrm{mV}$ (middle) $\tau_{w}=50 \mathrm{~ms}$ (right) $\tau_{d}=5 \mathrm{~ms}$ 


\subsubsection{Minimum connections and type II AdEx neurons}

As Neurons of different membrane excitability associated with different types of $f-I$ curve and corresponding to distinct neural dynamics, they consequently impact network synchrony. We point out that neurons in these networks are behaving like type I $f-I$ curve as shown in (Fig. 1). Hence, we explore $M_{\text {syn }}^{\min }$ for AdEx neuron of type II $f-I$ curve (referred as type II neurons thereafter). Fig. 14 (left) demonstrates that, AdEx neurons of type II, network synchrony is first unobserved then followed by a sudden onset of network synchrony which is network size dependent. The full-scale network synchrony is difficult to reach $(\kappa \neq 1)$. For large network sizes of $N=500$ and 1000, network synchrony increases relatively slowly, as $M_{\text {syn }}$ increases. Fig. 14 (right) indicates that $M_{s y n}^{\min }$ is network size dependent.
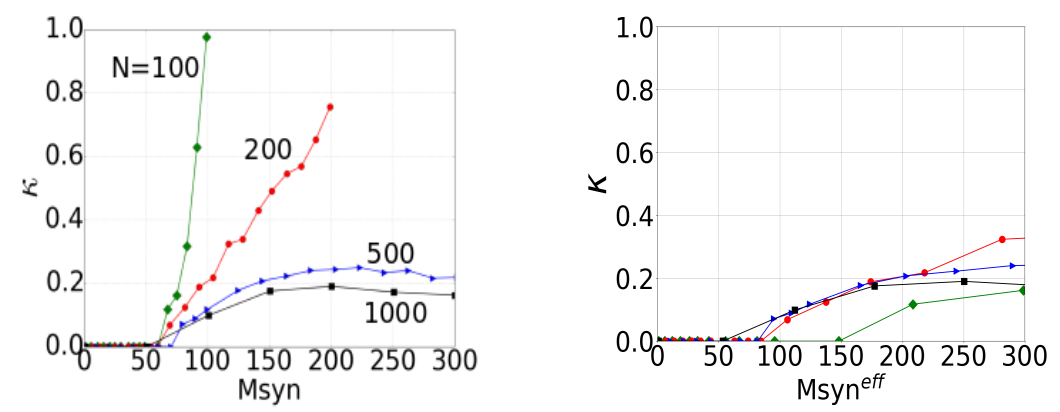

Figure 14: $\kappa$ vs $M_{\text {syn }}$ and $M_{\text {syn }}^{\text {eff }}$ plots for AdEx neurons of type II. (left) The onset of network synchrony is sudden and varied in each curve. (right) Normalized curves do not converge showing $M_{\text {syn }}^{\text {min }}$ being network size dependent.

\subsection{Izhikevich model and Morris-Lecar model}

\subsubsection{Minimum connections and Izhikevich neurons}

Due to similarities of the richness in neural dynamics and broad range of neural activity patterns in Izhikevich model $[27,28]$, we also study networks of Izhikevich neurons under the concept of determining $M_{s y n}^{\min }$. Fig. 15 (left) illustrates that $M_{s y n}^{\min } \approx 110$ for type I Izhikevich neurons with sufficient network sizes $N \geq 200$. For a smaller network size, $N=100$, it shows $M_{s y n}^{\min }$ is less than 110. Fig. 15 (right) further illustrates that all normalized curves converge into one before $M_{s y n}^{\text {eff }}$ reaching about 110, then diverge off afterwards. This indicates that though $M_{s y n}^{\min }$ remains about 110, to achieve network synchrony beyond the onset, $M_{s y n}^{\min }$ is network size dependent. Fig. 16 (left) illustrates that network synchrony is first unobserved then followed by a sudden onset of network synchrony for smaller network sizes $(N \leq 500)$, and $M_{s y n}^{\min }$ is generally low $(\approx 30)$ for type II Izhikevich neurons. Fig. 16 (right) indicates that, to achieve network synchrony beyond the onset, $M_{s y n}^{\min }$ is network size dependent. 

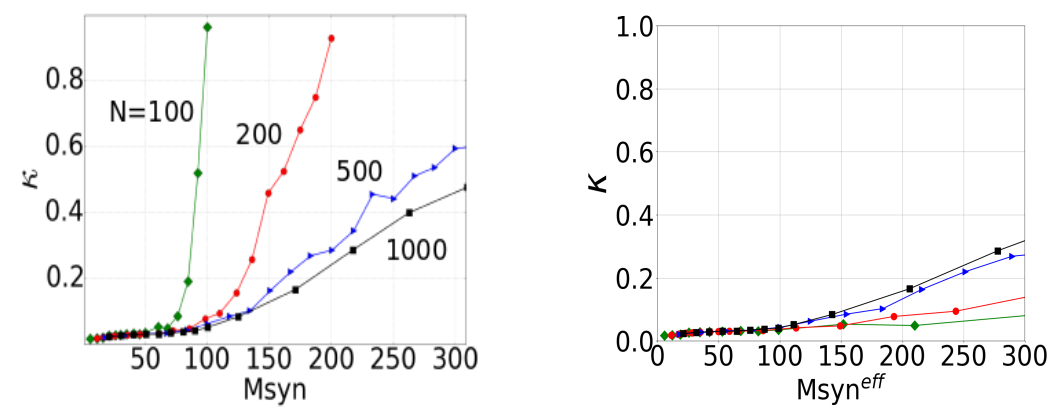

Figure 15: $\kappa$ vs $M_{\text {syn }}$ and $M_{\text {syn }}^{\text {eff }}$ plots for networks of $N$ Izhikevich neurons of type I. (left) $M_{\text {syn }}^{\min } \approx 110$ for $N \geq 200$. (right) All normalized curves converge to one until $M_{\text {syn }}^{e f f}$ reaching about 110 , then become separate afterwards $\left(I=34 \mathrm{pA}, g_{\text {syn }}=6 \mathrm{nS}\right)$.
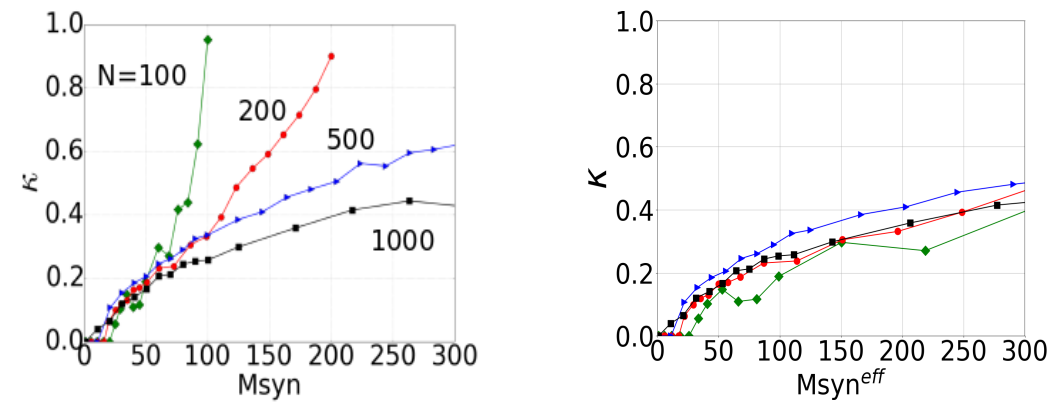

Figure 16: $\kappa$ vs $M_{\text {syn }}$ and $M_{\text {syn }}^{e f f}$ plots for networks of $N$ Izhikevich neurons of type II. (left) $M_{\text {syn }}^{\text {min }} \approx 30$. (right) Normalized curves do not converge $\left(I=15 \mathrm{pA}, g_{\text {syn }}=2 \mathrm{nS}\right)$.

\subsubsection{Minimum connections and Morris-Lecar neurons}

As a comparison to two-variable models of a different nature, such as a continuous model, we study networks of Morris-Lecar neurons under the concept of determining $M_{s y n}^{\min }$. Fig. 17 (left) illustrates that $M_{s y n}^{m i n}$ is about 200 for Morris-Lecar neurons of type I, and network synchrony grows only slightly beyond the onset followed by a full network synchrony at all-to-all connections. The normalized curves do not converge (Fig. 17 (right)), indicating that, to achieve network synchrony beyond the onset, $M_{\text {syn }}^{\min }$ is network size dependent.

The fact that the $f-I$ curve of Morris-Lecar model exhibits a slow rise frequency before damping down to zero with increasing currents may explain the network behavior difference between this model and other models presented here. Type II Morris-Lecar neurons exhibit similar behaviors to type I neurons, hence they are not shown.

In summary, Fig. 12 to Fig. 17 provide road maps for the minimum synaptic connections needed to build neuronal networks via these three models (of either types I or II neurons) to achieve expected network synchrony. These model simulations demonstrate 

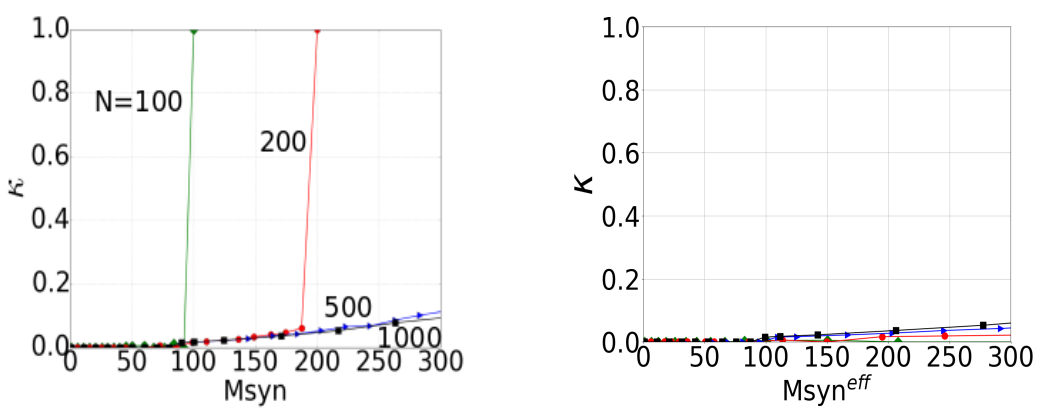

Figure 17: $\kappa$ vs $M_{\text {syn }}$ and $M_{\text {syn }}^{\text {eff }}$ plots for networks of $N$ Morris-Lecar neurons of type I. (left) Network synchrony is difficult to observe for small network, $N=100$, until allto-all connection $\left(M_{s y n}=100\right)$ is reached. For larger networks, $N \geq 200, M_{s y n}^{\min } \approx 200$. (right) Normalized curves do not converge.

that to generate interneuronal network gamma rhythms approximately $30 \mathrm{~Hz}$, adopting AdEx neurons behaving like type $I$ is an appropriate choice for studying sparse and random inhibitory networks found in hippocampal $\mathrm{C} A 1$ area, and more importantly the minimum connections needed in such networks are relatively independent of network size. We will focus the rest of our study on the AdEx model (more about this choice in the following section).

\subsection{Gamma rhythms in hippocampus via sparse and random networks}

We are now ready to build networks via sparse and random connections to generate gamma rhythms found in hippocampus, and explore parameter regimes for inducing such oscillations. Under the consideration of more realistic networks, one may take into account of intrinsic or extrinsic noise, or other coupling features, and it will bring the network synchrony down. Hence, with that in mind, we build networks with network synchrony higher than its onset. For $\kappa \approx 0.3$, we take a network size $N=1000$ and the minimum connections of $M_{\text {syn }}=200>M_{\text {syn }}^{\text {min }}=60(\kappa \approx 0.05)$ (see Fig. 12 (left)) reflecting a sparse connection with the probability as low as $p=0.2$. This network structure indeed demonstrates a more pronounced and strong network synchrony with network frequency within gamma rhythms. For this purpose under the same $N, M_{\text {syn }}$ and $p,(1)$ type II AdEx neuron would have only achieved $\kappa=0.18$, (2) type I Izhikevich neuron would have only achieved $\kappa=0.18$, (3) type II Izhikevich neuron would have achieved $\kappa=0.3$, and (4) type I/II Morris-Lecar neuron would have only achieved $\kappa=0.05$, and they are all network size dependent. Though type II Izhikevich neuron can also achieve similar network sychrony, it exhibits none convergent effectiveness of the $M_{\text {syn }}$ curves and possesses none physiological relevant parameters. Hence, we favor the AdEx nerron of type I in this study.

We further explore the range of parameters, such as both adaptations $a$ and $b$, adaptive 
time constant $\tau_{w}$, synaptic time constants $\tau_{r}$ and $\tau_{d}$, synaptic conductance $g_{s y n}$, and the mean input current $I_{\mu}$ to understand how network synchrony and frequency can be produced and maintained within gamma rhythms found in hippocampus. We include the considerations of gap junction and shunting inhibition in sparse and random connection networks, and discuss how they may affect network synchrony and frequency. We also validate our results with experimental recording data, and discuss networks of AdEx to induce theta-nested gamma rhythms.

Intrinsic properties affect the networks: In general, increasing mean input current $I_{\mu}$ simultaneously increases network frequency and decreases network synchrony (Fig. 18). In (Fig. 18 (left top/bottom)), we demonstrate that, for the same $a$, a higher mean input current $I_{\mu}$ induces higher gamma rhythms, and decreases the network synchrony accordingly. Similarly for $b$ in network frequency, however, it behaves differently in network synchrony. Once $b$ reaches an optimal value, network synchrony deteriorates to asynchrony (Fig. 18 (middle top/bottom)). For $\tau_{w}$, similarly to $a$ or $b$, a higher input mean current $I_{\mu}$ induces higher frequencies in gamma rhythm (Fig. 18 (right top/bottom)), but eventually decreases network synchrony. These plots show how variations of intrinsic properties can reflect network frequency and synchrony in generating gamma rhythms in hippocampus.
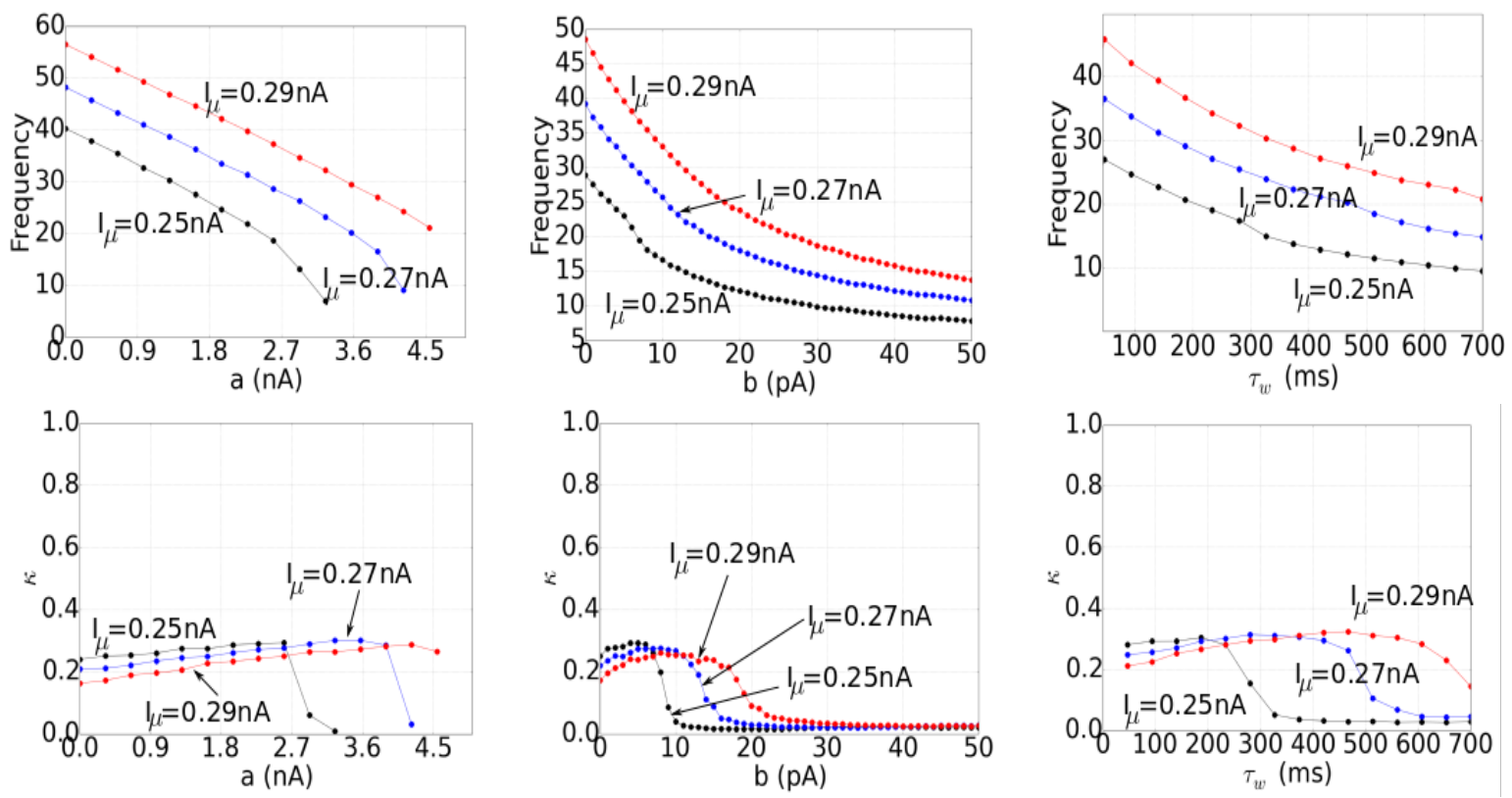

Figure 18: Frequency/synchrony vs $a, b$ and $\tau_{w}$; All three plots indicate that a higher input mean current $I_{\mu}$ leads to a higher network frequency (top row), and leads to a lower network synchrony before deteriorating to asynchrony (bottom row).

Synaptic properties affect the networks: In the aspects of synaptic input leading to network synchrony, the ranges of synaptic conductance $g_{\text {syn }}$ and the rising and decay 
time constants, $\tau_{r}$ and $\tau_{d}$, are demonstrated respectively. Similar to intrinsic properties, in general, increasing mean input current $I_{\mu}$ simultaneously increases network frequency and decreases network synchrony (Fig. 19). In (Fig. 19 (left top/bottom)), it is shown that network frequency increases while network synchrony decreases, as the maximaum $g_{s y n}$ increases. The variation of rising synaptic time constant $\tau_{r}$ is relatively insensitive to the network frequency and synchrony (Fig. 19 (middle top/bottom)). Similar to synaptic conductance $g_{\text {syn }}$, decay synaptic time constant $\tau_{d}$ impacts network frequency and synchrony more substantially (Fig. 19 (right top/bottom)). The plots suggest that there is an optimal decay time constant for each mean current input to produce higher gamma rhythms and maintain higher network synchrony. Once delay time constant passes that optimal value, both network frequency and synchrony deteriorate.
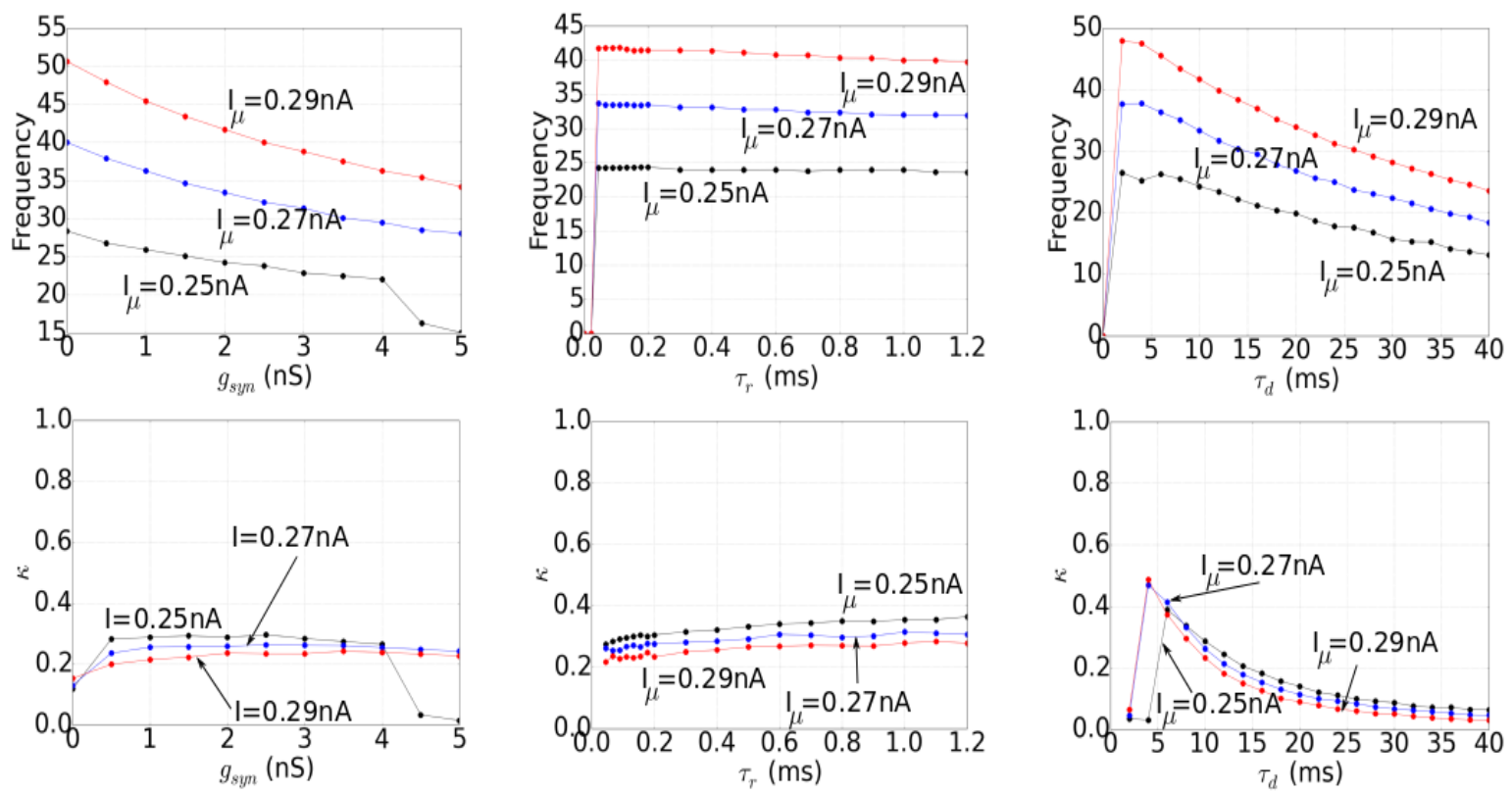

Figure 19: Frequency/synchrony plots: Increasing mean input current $I_{\mu}$ increases network frequency and decreases network synchrony. (top left) Stronger $g_{\text {syn }}$ decreases network frequency. (bottom left) Network synchrony appears to be insensitive to $g_{\text {syn }}$. (top middle) Network frequency nearly remains, as $\tau_{r}$ varies. (bottom middle) Increasing $\tau_{r}$ influences network synchrony slightly. (top right) When $\tau_{d}$ is small and at certain optimal value, network frequency achieves maximum. Once the value is exceeded, network frequency continues to decrease. (bottom right) Similarly, network synchrony decreases as $\tau_{d}$ passes an optimal value.

Optimal synchronization in gamma rhythms: Based on the demonstrations of how intrinsic and synaptic properties influence network frequency and synchrony via sparse random networks, we further demonstrate these networks exhibit a peak preferred frequency range (Fig. 20 (left)). It shows that network frequency about $25 \mathrm{~Hz}$ induces the peak network synchrony. As network frequency increases, the network synchrony 
decreases accordingly. Hence, varying synaptic conductance $g_{\text {syn }}$ within a small range exhibits a preferred network frequency of gamma rhythms while ensuring the degrees of network synchrony.
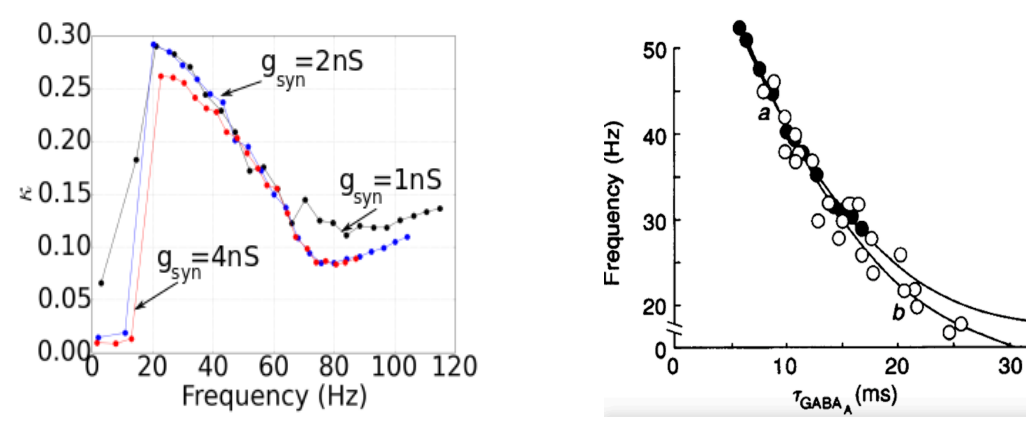

Figure 20: (left) All curves show a preference of peak network synchrony around $25 \mathrm{~Hz}$ (right) Network frequency responses to synaptic time constant of experimental recordings in rat hippocampus (circles) and model simulations (dots) in [48].

Experimental recording data: Experimental recordings of rodent by Traub et al. [48] show that interneuronal network of gamma rhythms in CA1 of the hippocampus can be induced in vitro during blockade of ionotropic glutamate receptors, and network frequency declines with synaptic time constant prolonged (Fig. 20 (right)). We compare the gamma network frequency generated by sparse randomly connected networks (Fig. 19 (top right)) with experimental recordings shown in (Fig. 20 (right)), and find that both network frequency responses to synaptic (decay) time constant are remarkably comparable.

Gap junctions: Studies have shown that electrical synapses among interneuronal networks may play an important but different role from chemical synapses in contributing to network synchrony [31]. Previously, we demonstrated that, in sparse randomly connected networks inducing gamma oscillations, stronger chemical synapses reduce network frequency and maintain similar network synchrony (Fig. 19 (left)). In contrast, with the inclusion of electrical synapses, the simulation demonstrates a strong enhancement in network synchrony while maintaining similar frequency (Fig.21). It indicates that electrical synapses can enhance network synchrony of gamma oscillations in sparse randomly connected interneuron networks rather effectively [47]. Our simulations demonstrate that, without electric synapses, network frequency is $24,33,42(\mathrm{~Hz})$ for $I=0.25,0.27,0.29(\mathrm{nA})$ with standard deviation $0.30,0.44,0.57(\mathrm{~Hz})$ respectively in sparse random networks. In contrast, with the inclusion of electric synapses of $g_{\text {gap }}=0.5$ $\mathrm{nS}$, though network frequency is preserved respectively, all standard deviations become zero. This indicates that interneurons with electrical synapses via AdEx model improve network synchrony significantly leading to greater robustness. This result is consistent with the finding that inhibitory synaptic coupling can increase the dispersion of neuronal voltages between spikes, whereas electrical coupling reduces such dispersion [31]. 

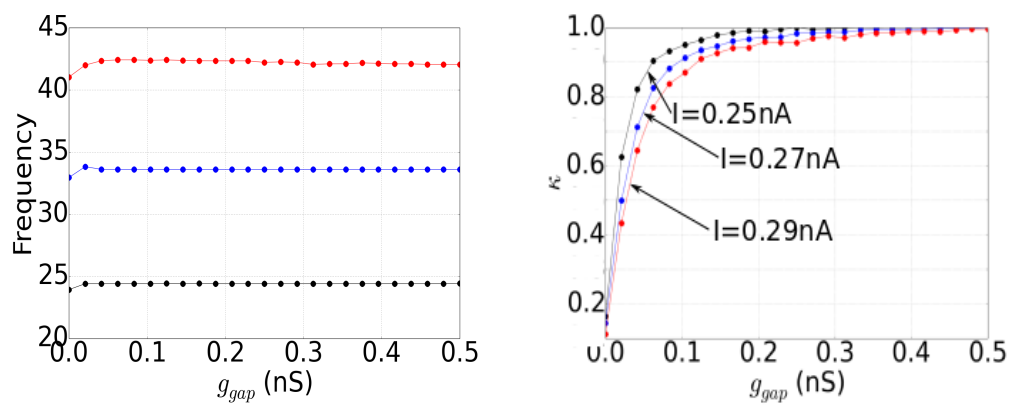

Figure 21: Stronger gap junction maintains similar network frequency (left), but enhances network synchrony significantly (right).

Shunting inhibition: When shunting inhibition is included [50] in sparse random networks, network frequency slows down (Fig.22), while network synchrony remains relatively the same. Previous study suggests that shunting inhibition may enhance gamma oscillations with greater robustness [50]. Our simulations demonstrate that, without shunting inhibition, network frequency is $24,33,42(\mathrm{~Hz})$ for $I=0.25,0.27,0.29(\mathrm{nA})$ with standard deviation $0.30,0.44,0.57(\mathrm{~Hz})$ respectively in sparse random networks. With the inclusion of shunting inhibition of $g_{\text {shunt }}=1 \mathrm{nS}$, network frequency reduces to $19,29,37(\mathrm{~Hz})$ with standard deviation increased to $0.32,0.45,0.53(\mathrm{~Hz})$ respectively in sparse random networks. This indicates that interneurons with shunting inhibitions via AdEx model lower network frequency and increase dispersion of network frequency. (which behaves similar to $\mathrm{H}-\mathrm{H}$ type neuron as in [53]).
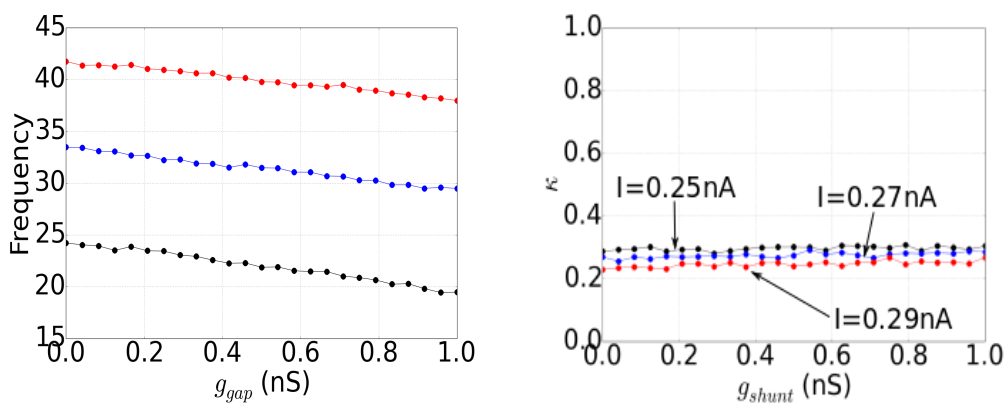

Figure 22: Stronger shunting inhibition (left) reduces network frequency, and (right) keeps network synchrony relatively constant (but increase the frequency dispersion).

Theta-nested gamma rhythms: A particularly prominent rhythmic pattern is that of oscillations exhibit nested activity at theta and gamma frequencies seen under conditions of active exploration in the rat hippocampal formation. It is believed to be a set of structures necessary for declarative memory $[57,5]$. Gamma rhythms may be generated locally by interactions within a class of interneurons mediating fast $\mathrm{GABA}_{\mathrm{A}}\left(\mathrm{GABA}_{\mathrm{A}, \text { fast }}\right)$ inhibitory post-synaptic currents. Recent evidence indicates that a slower class of $\mathrm{GABA}_{\mathrm{A}}\left(\mathrm{GABA}_{\mathrm{A} \text {,slow }}\right)$ interneurons in the hippocampus projects to the $\mathrm{GABA}_{\mathrm{A} \text {,fast }}$ interneurons that contribute to hippocampal mixed theta-gamma rhythms $[57,5]$. These 
two synaptic responses, $\mathrm{GABA}_{\mathrm{A} \text {,fast }}$ and $\mathrm{GABA}_{\mathrm{A} \text {,slow }}$, appear to arise from distinct populations of interneurons. In much the same way that $\mathrm{GABA}_{\mathrm{A} \text {,fast }}$ cells seem to control the gamma rhythm, and $\mathrm{GABA}_{\mathrm{A} \text {,slow }}$ cells represent a prime candidate mechanism, wholly within the hippocampal formation, that may contribute to the theta rhythm. We use AdEx models coupling populations of $\mathrm{GABA}_{\mathrm{A} \text {,fast }}$ and $\mathrm{GABA}_{\mathrm{A} \text {,slow }}$ interneurons via network of Fig. 23 (left) for generating nested theta and gamma rhythms in region CA1 of the hippocampus. In (Fig.23 (right)), it demonstrates that the population of slow $\mathrm{GABA}_{\mathrm{A}}$ interneurons oscillate in theta rhythm and the population of fast $\mathrm{GABA}_{\mathrm{A}}$ interneurons oscillate in gamma rhythm to induce a theta-nested gamma rhythm reflecting the active responses found in rat hippocampus.
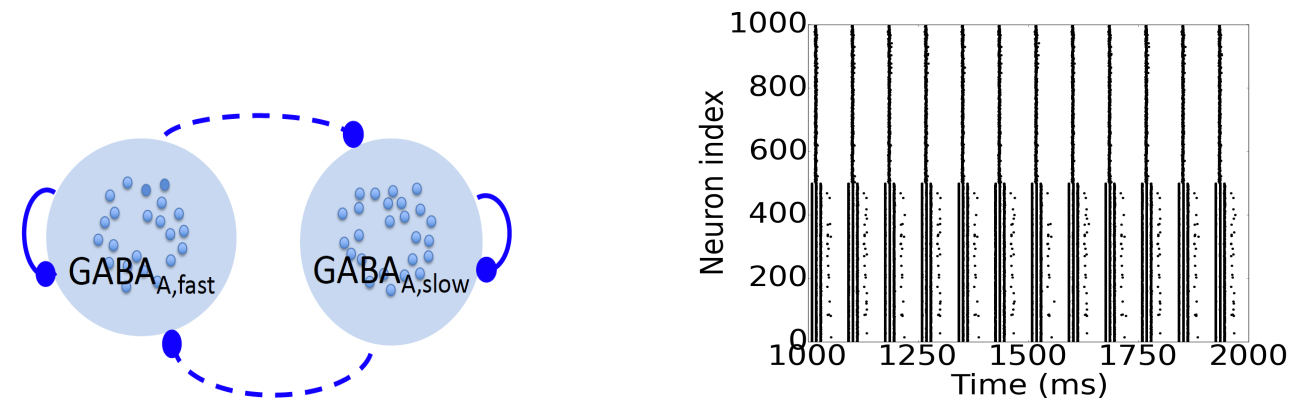

Figure 23: (left) Two populations of interneuronal circuity in region CA1 for thetanested gamma oscillations; (right) A population of $500 \mathrm{GABA}_{\mathrm{A} \text {,slow }}$ interneurons oscillate in theta band $(\approx 10 \mathrm{~Hz})$ and a population of $500 \mathrm{GABA}_{\mathrm{A} \text {,fast }}$ interneurons oscillate in gamma band $(\approx 30 \mathrm{~Hz})$. Random connection probability $p=0.2$; synaptic peak conductance: $g_{\text {syn (fast, fast })}=0.0307 \mathrm{nS}, g_{\text {syn }(\text { fast,slow })}=0.001 \mathrm{nS}, g_{\operatorname{syn}(\text { slow,fast })}=0.002 \mathrm{nS}$, $g_{\text {syn }(\text { slow, slow })}=0.15 \mathrm{nS}$; synaptic rise and decay time constants: $\tau_{r \text { (fast) }}=0.1 \mathrm{~ms}, \tau_{d(\text { fast })}=$ $10 \mathrm{~ms}, \tau_{r \text { (slow) }}=5 \mathrm{~ms}, \tau_{d \text { (slow) }}=50 \mathrm{~ms}$; sinusoidal input current at theta band: $I=0.28$ $\mathrm{nA}+0.15 \sin (24 \pi t) \mathrm{nA}$

\subsection{Discussion and conclusions}

Gamma rhythms in the brain may be induced through three network mechanisms, such as pure interneurons, an interplay between interneurons and pyramidal neurons, and a weak participation of pyramidal neurons with interneurons. Among them, networks of interneurons are the most significant and fundamental mechanism to induce gamma band activities, and it is therefore the one focused in this study. With this mechanism, though frequencies of individual neurons are higher than the network frequency, they are near the network frequency. Our findings in this study shows persistence with this property. Ultimately interneuron networks are generally embedded in larger networks with a participation of pyramidal neurons, and it will be of interest to investigate the interplay of interneurons and pyramidal neurons or the more complex network with a weak pyramidal participation to generate gamma rhythms.

AdEx neuronal networks coupled with AMPA-mediated type excitatory synapses fail 
to synchronize as expected, whereas GABAergic inhibitory coupling leads to collective activities in gamma band. This result is in agreement with the previous studies in the same direction, such as $[53,24,51]$ on excitatory networks, $[53,55,56,51]$ on inhibitory networks, and $[53,2,61,48]$ on the generation of interneuron gamma rhythms. We examine the emergence of gamma rhythms from networks which can be observed in neocortex and in hippocampus during behavioral arousal through AdEx neuron, and identify the following necessary conditions for such network synchrony via GABAergic synaptic coupling. (1) For single neurons, the subthreshold and spike-triggered adaptations need to be small to maintain gamma rhythms, the time constant of neuronal adaptation current $\left(\tau_{w}\right)$ is less sensitive by allowing a wide range to show gamma rhythms, and the hyperpolarization after a spike should be above the synaptic reversal potential to ensure the synaptic inputs being hyperpolarizing. (2) As a synaptic property, the synaptic decay should be relatively slow such that the ratio between the synaptic decay time constant and the spiking period is not too small, to observe the effects of synaptic inputs. (3) As a network property, the heterogeneities of input currents should be adequately small to assure network synchrony. In addition, random and heterogenous networks based on these properties, shows a minimum number of connections per neuron necessary to generate network synchrony, and this number is weakly dependent on the network size. Though subthreshold and spike-triggered adaptation features are unique to neurons equipped with adaptation parameters such as AdEx model, they are prevalent in biological neurons and known to slow down network frequency similar to those observed in condition (1). Time constant in synaptic rising is generally fast compared to its slow decay as shown in conditions (2). However, the slower synaptic decay induces slower network frequency. Interneuronal network gamma rhythms are often fragile against heterogeneity and noise, such as in interneuron gamma oscillation studies $[53,56,6]$ and experimental recording [48], and this result is consistent with our study stated in condition (3).

Electrical and chemical synapses both exist within the same interneuron networks, and each type of synapse is able to promote network synchrony through playing different roles. In interneuron networks of gamma rhythms, the $\gamma$-aminobutyric acid type A, $\mathrm{GABA}_{\mathrm{A}}$, mediated inhibition is relatively strong. Under this condition, addition of a small electrical conductance can increase the degree of network synchrony far more than a much larger increase in inhibitory conductance. The electrical synapses increase network synchrony through suppressing the heterogeneity in the network. Hence, electrical synapses significantly improve network synchrony and robustness. It is shown that $\mathrm{GABA}_{\mathrm{A}}$ mediated inhibition in mature interneurons of the hippocampus demonstrate gyrus is shunting rather than hyperpolarizing. Nevertheless, when shunting inhibition is incorporated into our study, network synchrony barely improved. It indicates that AdEx model does not reflect well with mature interneurons of the hippocampus to generate gamma rhythms.

Meanwhile, it was thought that, with more biophysically realistic models, less neurons maybe needed to observe network synchrony than with less biophysically detailed models. Nevertheless, the results in our study shows remarkable similarity to those of $\mathrm{H}-\mathrm{H}$ type neuron study shown in [53]. We provide a significant road map to work out 
the minimum synaptic connections needed in order to achieve any desired degree of network synchrony according to the network size for AdEx model. The existence of a minimum number of connections necessary to generate network synchrony has also been found in other network structures $[1,54]$, indicating that this weak-dependence may be a general feature of sparsely connected and random networks. While type I Izhikevich model may do well in synchronizing network activities, it is network size sensitive and of less biophysical relevance.

Rhythms within a neuronal network can be generated by local mechanisms, driven by remote inputs, or induced by a combination of extrinsic and intrinsic properties. We use new evidence for connections from $\mathrm{GABA}_{\mathrm{A} \text {,slow }}$ cells to the more well-known $\mathrm{GABA}_{\mathrm{A} \text {,fast }}$ interneurons to create a network based on two populations of inhibitory neurons. The network is able to autonomously create the nested theta-gamma rhythm observed in hippocampus, under the conditions of strong connections among population, weaker connections between populations, and carefully tuned input drives. This indicates that the fragility of nested theta-gamma rhythms induced through such network structures may be improved, possibly via the inclusion of pyramidal neurons or synaptic delays.

This study demonstrates that a low complexity AdEx model performs similarly to and can do as well as $\mathrm{H}-\mathrm{H}$ type model type model. This provides researchers a novel mean of studying various rhythmic activities found in hippocampus through a biophysical relevant and low dimensional neuron model.

\subsection{Acknowledgments}

We thank Xiao-Jing Wang for the valuable discussion, and Kanishka Basnayake for his input. LS is supported by National Science Foundation (DMS-1226282).

\section{References}

[1] K. I. Barkai, E. and H. Sompolinsky. Properties of sparsely connected excitatory neural networks. Phys Rev A, 41:590-597, 1990.

[2] E. Bragin, G. Jando, Z. Nadasdy, J. Hetke, K. Wise, and G. Buzsáki. Gamma (40$100 \mathrm{hz}$ ) oscillation in the hippocampus of the behaving rat. J Neurosci, 15:47-60, 1995.

[3] R. Brette and W. Gerstner. Adaptive Exponential Integrate-and-Fire model as an effective description of neuronal activity. J. Neurophysiol., 94(5):3637, 2005.

[4] N. Brunel. Phase diagrams of sparsely connected networks of excitatory and inhibitory spiking neurons. Neurocomputing, 32-33:307-312, 2000.

[5] G. Buzsaki. Rhythms of the Brain. Oxford University Press, 2006. 
[6] Y. Cao, K. S. Wilcox, C. E. Martin, E. J. Rachinsky, T. L., and M. A. Dichter. Presence of mRNA for glutamic acid decarboxylase in both excitatory and inhibitory neurons. Neurobiology, 93:9844-9849, 1996.

[7] D. B. Chklovskii, B. W. Mel, and K. Svoboda. Cortical rewiring and information storage. Nature, 431(7010):782-788, 2004.

[8] C. Chow. Phase-locking in weakly heterogeneous neuronal networks. Physica D, 118:343-370, 1998.

[9] C. Clopath, R. Jolivet, A. Rauch, H. Luscher, and W. Gerstner. Predicting neuronal activity with simple models of the threshold type: Adaptive Exponential Integrateand-Fire model with two compartments. Neurocomputing, 70(10-12):1668, 2007.

[10] S. M. Crook, G. B. Ermentrout, and J. M. Bower. Spike frequency adaptation affects the synchronization properties of networks of cortical oscillations. Neural Computation, 10(4):837-854, 1998.

[11] S. M. Doesburg, A. B. Roggeveen, K. Kitajo, and L. M. Ward. Large-scale Gammaband phase synchronization and selective attention. Cerebral Cortex, 18(2):386-96, Mar. 2008.

[12] B. Ermentrout. Type I neurons, phase resettings curves and synchrony. Neural Comput, 8(5):979-1001, 1996.

[13] B. Ermentrout and T.-W. Ko. Delays and weakly coupled neuronal oscillators. Phil Trans Soc A, 367(1891):1097-115, 2009.

[14] B. Ermentrout, M. Pascal, and B. Gutkin. The effects of spike frequency adaptation and negative feedback on the synchronization of neural oscillators. Neural Comput, 13(6):1285-310, June 2001.

[15] G. B. Ermentrout and D. H. Terman. Mathematical Foundations of Neuroscience. New York, NY: Springer, 2010.

[16] N. Fourcaud-Trocmé, D. Hansel, C. van Vreeswijk, and N. Brunel. How spike generation mechanisms determine the neuronal response to fluctuating inputs. $J$. Neurosci., 23:11628, 2003.

[17] P. Fries, J. Reynolds, A. Rorie, and R. Desimone. Modulation of oscillatory neuronal synchronization by selective visual attention. Science, 291(5508):1560-1563, Feb. 2001.

[18] W. Gerstner. Population dynamics of spiking neurons: fast transients, asynchronous states, and locking. Neural Comput., 12(1):43-89, 2000.

[19] W. Gerstner, J. L. Van Hemmen, and J. D. Cowan. What matters in neuronal locking? Neural Comput., 8:1653-1676, 1996. 
[20] D. Golomb and D. Hansel. The number of synaptic inputs and the synchrony of large, sparse neuronal networks. Neural Computation, 12(5):1095-1139, 1999.

[21] D. F. M. Goodman and R. Brette. The Brian simulator. Frontiers in Neuroscience, 3:192-197, 2009.

[22] C. M. Gray. Synchronous oscillations in neuronal systems: Mechanisms and functions. J. Comp. Neurosci., 1:11-38, 1994.

[23] D. Hansel, G. Mato, and C. Meunier. Synchrony in excitatory neural networks. Neural Computation, 7(2):307-337, 1995.

[24] C. Herrmann, M. Munk, and A. Engel. Cognitive functions of gamma-band activity: memory match and utilization. Trends Cogn Sci, 8(8):347-55, 2004.

[25] J. Hipp, A. Engel, and M. Siegel. Oscillatory synchronization in large-scale cortical networks predicts perception. Neuron, 69(2):387-396, 2011.

[26] E. Izhikevich. Simple model of spiking neurons. IEEE Trans Neural Netw, 14(6):1569-72, 2003.

[27] E. Izhikevich. Dynamical Systems in Neuroscience: The geometry of excitability and bursting. the MIT press, Massachusetts, 2007.

[28] R. Jolivet, F. Schürmann, T. K. Berger, R. Naud, W. Gerstner, and A. Roth. The quantitative single-neuron modeling competition. Biol. Cybern., 99(4-5):417, 2008.

[29] N. Kopell. Toward a theory of modeling central pattern generators. In A. Cohen (Ed.). New York: Wiley, 1988.

[30] N. Kopell and G. Ermentrout. Chemical and electrical synapses perform complementary roles in the synchronization of interneuronal networks. Proc. Nat. Acad. Sci. U.S.A., 101:15482-7, 2004.

[31] J. Ladenbauer, M. Augustin, L. Shiau, and K. Obermayer. Impact of adaptation currents on synchronization of coupled exponential integrate-and-fire neurons. PLoS Comput. Biol., 8(4), 2012.

[32] S. Lefort, C. Tomm, J. C. Floyd Sarria, and C. C. H. Petersen. The excitatory neuronal network of the $\mathrm{c} 2$ barrel column in mouse primary somatosensory cortex. Neuron, 61(2):301-316, 2009.

[33] M. Lengyel, J. Kwag, O. Paulsen, and P. Dayan. Matching storage and recall: hippocampal spike timing-dependent plasticity and phase response curves. Nature neurosci, 8(12):1677-83, 2005.

[34] J. W. Lichtman and J. R. Sanes. Ome sweet ome: what can the genome tell us about the connectome? Current Opinion in Neurobiology, 18(3):346-353, 2008. 
[35] L. Melloni, C. Molina, M. Pena, D. Torres, W. Singer, and E. Rodriguez. Synchronization of neural activity across cortical areas correlates with conscious perception. J Neurosci, 27(11):2858-65, 2007.

[36] L. Neltner, D. Hansel, G. Mato, and C. Meunier. Synchrony in heterogeneous networks of spiking neurons. Neural computation, 12(7):1607-1641, 2000.

[37] A. N. Pisarchik, R. Meucci, F. T. Arecchi, E. Allaria, a. Di Garbo, J. Kurths, E. Rosa, D. Hansel, G. Mato, w. D. C. Boccaletti, S., J. A. Yorke, M. a. Wilson, B. L. McNaughton, R. Tokunaga, T. S. City, U. Ernst, I. Tokuda, S. Kajiwara, T. Matsumoto, et al., and van Vreeswijk C. Dynamics of sparsely connected networks of excitatory and inhibitory spiking neurons. Phys. Rev. Let., 86(1):41754178, 2001.

[38] H. S. Seung. Reading the book of memory: Sparse sampling versus dense mapping of connectomes. Neuron, 62(1):17-29, 2009.

[39] W. Singer. Neuronal synchrony: A versatile code for the definition of relations? Neuron, 24:49-65, 1999.

[40] S. Song, P. J. Sjstrm, M. Reigl, S. Nelson, and D. B. Chklovskii. Highly nonrandom features of synaptic connectivity in local cortical circuits. PLoS Biology, 3(3):e68, 2005 .

[41] A. Stepanyants, J. A. Hirsch, L. M. Martinez, Z. F. Kisvrday, A. S. Ferecsk, and D. B. Chklovskii. Local potential connectivity in cat primary visual cortex. Cerebral Cortex, 18(1):13-28, 2008.

[42] M. Stimberg, D. F. M. Goodman, V. Benichoux, and R. Brette. Equation-oriented specification of neural models for simulations. Frontiers in Neuroinformatics, 8(6), 2014.

[43] P. Tiesinga and T. J. Sejnowski. Cortical enlightenment: Are attentional gamma oscillations driven by ING or PING? Neuron, 63(6):727-732, 2009.

[44] R. A. Tikidji-Hamburyan, J. J. Martínez, J. A. White, and C. C. Canavier. Resonant interneurons can increase robustness of gamma oscillations. The Journal of Neuroscience, 35(47):15682-15695, 2015.

[45] J. Touboul and R. Brette. Dynamics and bifurcations of the adaptive exponential Integrate-and-Fire model. Biol. Cybern., 99(4-5):319, 2008.

[46] R. D. Traub, N. Kopell, A. Bibbig, E. H. Buhl, F. E. N. LeBeau, and M. A. Whittington. Gap junctions between interneuron dendrites can enhance synchrony of gamma oscillations in distributed networks. J. of Neuroscience, 21(23):94789486, 2001. 
[47] R. D. Traub, M. A. Whittington, S. B. Colling, G. Buzsaki, and J. G. Jefferys. Analysis of gamma rhythms in the rat hippocampus in vitro and in vivo. The Journal of Physiology, 493:471-484, 1996.

[48] A. Treves. Mean-field analysis of neuronal spike dynamics. Network, 4(3):259, 1993.

[49] I. Vida, M. Bartos, and P. Jonas. Shunting inhibition improves robustness of gamma oscillations in hippocampal interneuron networks by homogenizing firing rates. $\mathrm{Neu}$ ron, 49:107-117, 2006.

[50] V. Vreeswijk, L. Abbott, and B. Ermentrout. When inhibition not excitation synchronizes neural firing. J Comput Neurosci, 1(4):313-322, 1995.

[51] X.-J. Wang. Neurophysiological and computational principles of cortical rhythms in cognition. Physiol Rev, 90(3):1195 - 1268, 2010.

[52] X.-J. Wang and G. Buzsáki. Gamma oscillation by synaptic inhibition in a hippocampal interneuronal network model. J. Neurophysiol., 16(20):6402-13, 1996.

[53] X. J. Wang, D. Golomb, and J. Rinzel. Emergent spindle oscillations and intermittent burst firing in a thalamic model: specific neuronal mechanisms. Proc. Natl. Acad. Sci., 92(12):5577-5581, 1995.

[54] X. J. Wang and J. Rinzel. Alternating and synchronous rhythms in reciprocally inhibitory model neurons. Neural Comput, 4:84-97, 1992.

[55] X. J. Wang and J. Rinzel. Spindle rhythmicity in the reticularis thalami nucleus: synchronization among mutually inhibitory neurons. Neuroscience, 53:899-904, 1993.

[56] J. A. White, M. Banks, R. Pearce, and N. Kopell. Networks of interneurons with fast and slow $\gamma$-aminobutyric acid type a (gabaa) kinetics provide substrate for mixed gamma-theta rhythm. Proceedings of the National Academy of Sciences, 97(14):8128-8133, 2000.

[57] M. A. Whittington, M. O. Cunningham, F. E. LeBeau, C. Racca, and R. Traub. Multiple origins of the cortical gamma rhythm. Developmental Neurobiology, 71(1):92-106, 2010.

[58] M. A. Whittington, R. Traub, N. Kopell, B. Ermentrout, and E. Buhl. Inhibitionbased rhythms: experimental and mathematical observations on network dynamics. International journal of psychophysiology, 38(3):315-336, 2000.

[59] M. A. Whittington, R. D. Traub, and J. G. Jefferys. Synchronized oscillations in interneuron networks driven by metabotropic glutamate receptor activation. Nature, 373(6515):612-615, 1995. 